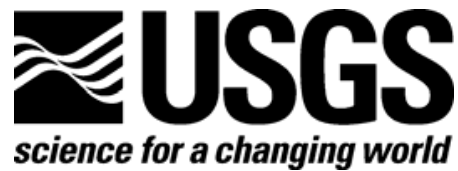

\title{
Physical and Chemical Data from Eolian Sediment Collected along a Transect from the Mojave Desert to the Colorado Plateau
}

By Harland L. Goldstein, Richard L. Reynolds, Marith C. Reheis, James C. Yount, and Paul J. Lamothe

Open-File Report 2007-1153

U.S. Department of the Interior

U.S. Geological Survey 


\section{U.S. Department of the Interior \\ DIRK KEMPTHORNE, Secretary}

\section{U.S. Geological Survey \\ Mark D. Myers, Director}

U.S. Geological Survey, Reston, Virginia 2007

For product and ordering information:

World Wide Web: http://www.usgs.gov/pubprod

Telephone: 1-888-ASK-USGS

For more information on the USGS—the Federal source for science about the Earth, its natural and living resources, natural hazards, and the environment:

World Wide Web: http://www.usgs.gov

Telephone: 1-888-ASK-USGS

Any use of trade, product, or firm names is for descriptive purposes only and does not imply endorsement by the U.S. Government.

This report has not been reviewed for stratigraphic nomenclature.

Although this report is in the public domain, permission must be secured from the individual copyright owners to reproduce any copyrighted material contained within this report. 


\section{Contents}

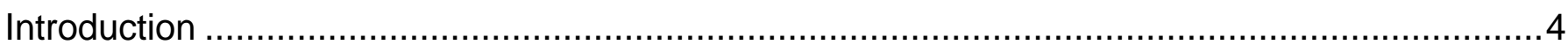

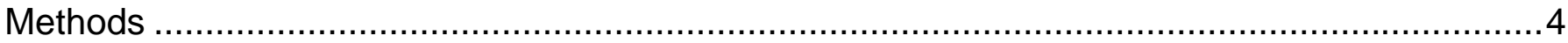

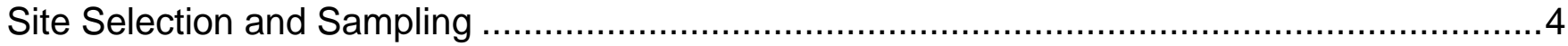

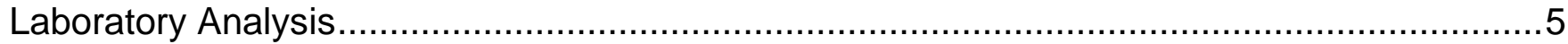

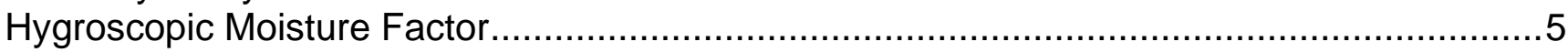

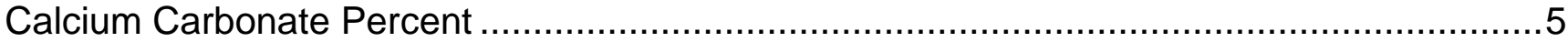

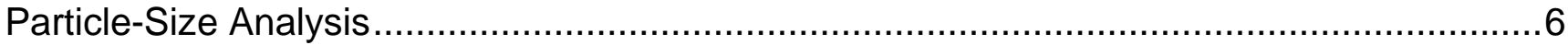

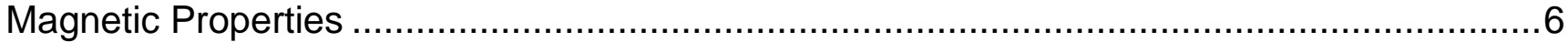

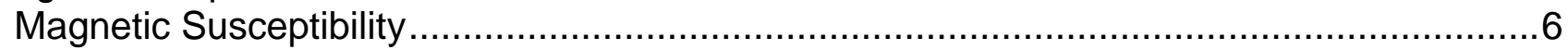

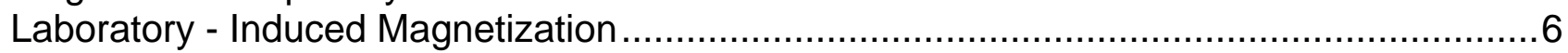

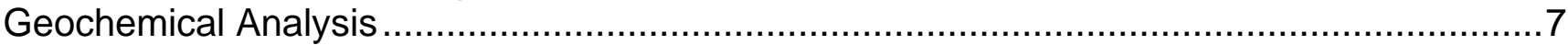

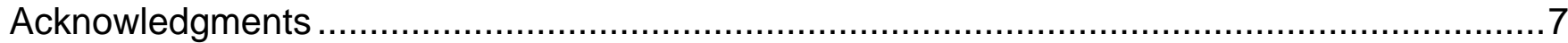

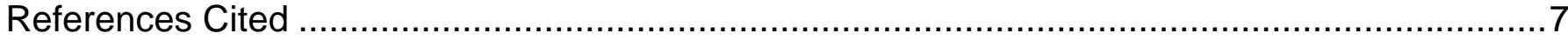

\section{Figures}

1. Generalized geologic map of the Southwestern United States showing the southwestnortheast transect of sampling sites.

\section{Tables}

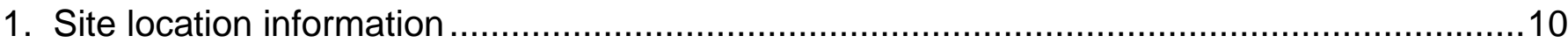

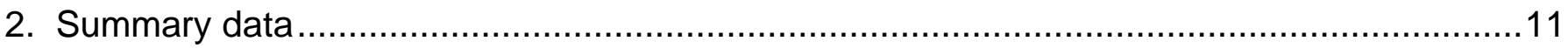

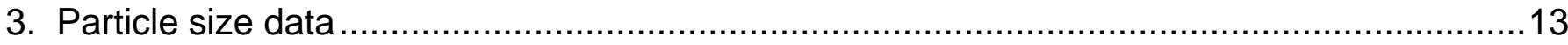

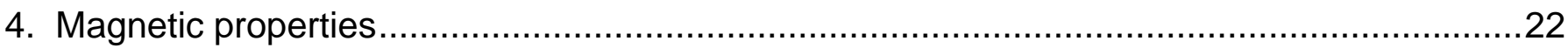

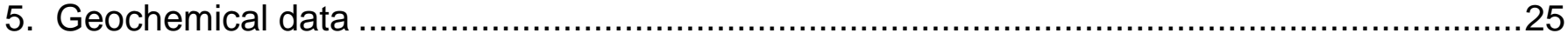




\section{Physical and Chemical Data from Eolian Sediment Collected along a Transect from the Mojave Desert to the Colorado Plateau}

By Harland L. Goldstein, Richard L. Reynolds, Marith C. Reheis, James C. Yount, and Paul J. Lamothe

\section{Introduction}

This report presents data and describes the methodology for magnetic, geochemical, and textural measurements of sediment and bedrock samples collected along a transect across the Southwestern United States (fig. 1).

The results presented here support a study that examines compositional variations of mineral dust deposited during the past few centuries in isolated natural traps spanning a region from the Mojave Desert of southern California to the central Colorado Plateau (Goldstein and others, in press; fig. 1). In particular, the study addresses the spatial and temporal variations in dust composition in the context of landscape geochemistry over a large area of the southwestern United States.

\section{Methods}

\section{Site Selection and Sampling}

The sampling sites were determined on the basis of spatial coverage along the transect and occurrences of mature eolian sandstone substrates. The sediments for this study were collected from natural depressions (potholes) that weathered into mostly eolian sandstones of Jurassic age (table 1). The sampled potholes are topographically isolated, so sediment in them can come from only two sources - airborne dust and detritus derived from the immediately surrounding catchment of the sandstone surface. The surface area of individual potholes and their catchments ranged from about 0.5 to $1.0 \mathrm{~m}^{2}$ and 50 to $100 \mathrm{~m}^{2}$, respectively.

Sediment in each pothole was sampled incrementally by depth. Three sediment samples from most potholes were collected with a trowel at depths of $0-1 \mathrm{~cm}, 1-2 \mathrm{~cm}$, and $2-5 \mathrm{~cm}$. Only 
two sediment samples, $0-1 \mathrm{~cm}$ and $1-2 \mathrm{~cm}$, were collected from site $05 \mathrm{U}-1$. For each sample, about 50 grams of fine-grained material was obtained over an area of $0.5-1.0 \mathrm{~m}^{2}$. A sample of the bedrock in which the pothole formed was also collected. Pothole elevations were determined from 30-meter digital elevation models (National Elevation Dataset, http://seamless.usgs.gov).

\section{Laboratory Analysis}

Samples were analyzed at the USGS Earth Surface Processes soils and magnetics laboratories (Denver, Colo.) for hygroscopic moisture factor, calcium carbonate percent, particle size, and magnetic properties (table 2). The soil analyses followed the protocols outlined in Singer and Janitzky (1986). Geochemical analysis was performed by the U.S. Geological Survey Geologic Discipline geochemistry laboratoy (Denver, Colo.).

\section{Hygroscopic Moisture Factor}

The hygroscopic moisture factor (table 2) is a measure of the moisture content of a sample at or near field conditions and is calculated by:

$\mathrm{H}=\mathrm{OD} / \mathrm{AD}$, where

$\mathrm{H}=$ hygroscopic moisture factor

$\mathrm{OD}=$ oven-dry sample weight $\left(105^{\circ} \mathrm{C} ; 16\right.$ hours $)$

$\mathrm{AD}=$ air-dry sample weight

\section{Calcium Carbonate Percent}

Calcium carbonate percent (table 2) was measured using a Chittick apparatus (Dreimanis, 1962) as described by Machette (1986), whereby $6 \mathrm{~N} \mathrm{HCl}$ is applied to the sample and the gas evolved from the reaction displaces fluid within the Chittick apparatus. The volume of displaced liquid is used to calculate the percentage of calcium carbonate in the sample.

$\mathrm{CaCO}_{3} \%=\left(\mathrm{VCO}_{2} * \mathrm{C}\right) / \mathrm{WS}$, where

$\mathrm{CaCO}_{3} \%=$ calcium carbonate percent

$\mathrm{VCO}_{2}=$ The volume of liquid displaced during reaction in presence of $\mathrm{HCl}$

$\mathrm{C}=$ Variable; $(\mathrm{PB} / \mathrm{TK}) * 0.16$, where

$\mathrm{PB}=$ barometric pressure in $\mathrm{Hg} \mathrm{mm}$

$\mathrm{TK}=$ temperature in Kelvin

WS $=$ oven-dry sample weight $\left(105^{\circ} \mathrm{C} ; 16\right.$ hours $)$ 


\section{Particle-Size Analysis}

Particle-size analysis (PSA) was performed on the $<2$-mm-size fraction of the pothole sediment and on bulk bedrock by using a laser analyzer (tables 2 and 3). Bedrock samples were lightly disaggregated to obtain the PSA sample. Prior to analysis, all samples were prepared by digesting organic matter and $\mathrm{CaCO}_{3}$ using 30 percent $\mathrm{H}_{2} \mathrm{O}_{2}$ and 15 percent $\mathrm{HCl}$, respectively. Also prior to analysis, sodium hexametaphosphate was added to each sample to deflocculate clays.

\section{Magnetic Properties}

Magnetic properties were determined on both $<2$-mm- and $<63$ - $\mu$ m-size fractions of the pothole samples as well as on bulk bedrock samples (tables 2 and 4). Magnetic properties determined included low-frequency and high-frequency magnetic susceptibility $\left(\mathrm{MS}_{\mathrm{lf}}, \mathrm{MS}_{\mathrm{hf}}\right)$, anhysteretic remanent magnetization (ARM), and isothermal remanent magnetization (IRM).

\section{Magnetic Susceptibility}

Magnetic susceptibility (MS) was measured by using a susceptometer with a sensitivity better than about $4 \times 10^{-7} \mathrm{~m}^{3} / \mathrm{kg}$. Samples were measured in a $0.1-\mathrm{mT}$ (millitesla) induction at a low frequency of $600 \mathrm{~Hz}\left(\mathrm{MS}_{\mathrm{lf}}\right)$ and high frequency of $6,000 \mathrm{~Hz}\left(\mathrm{MS}_{\mathrm{hf}}\right)$. For each sample, the MS value was determined as the mean of four measurements. Frequency-dependent susceptibility was calculated as:

$$
\mathrm{FDMS}=\left(\mathrm{MS}_{\mathrm{lf}}-\mathrm{MS}_{\mathrm{hf}}\right) / \mathrm{MS}_{\mathrm{lf}} .
$$

\section{Laboratory-Induced Magnetization}

Measurements of anhysteretic remanent magnetization (ARM) and isothermal remanent magnetization (IRM) experiments were made by using a high-speed spinner magnetometer. Anhysteretic remanent magnetization was imparted in a decreasing alternating field from a peak induction of $100 \mathrm{mT}$ and a DC bias of $0.1 \mathrm{mT}$. IRM magnetizations were generated at room temperature by using an impulse magnetizer. First, IRM was imparted in a 1.2-T induction $\left(\mathrm{IRM}_{1.2 \mathrm{~T}}\right)$. The samples then were magnetized in the opposite direction by using an induction of $0.3 \mathrm{~T}$ (IRM-0.3T). Hard isothermal remanent magnetization (HIRM) and the S-parameter were calculated as follows (King and Channel, 1991):

$$
\mathrm{HIRM}=\left(\operatorname{IRM}_{1.2 \mathrm{~T}}+\operatorname{IRM}_{-0.3 \mathrm{~T}}\right) / 2 \text {; }
$$




$$
\mathrm{S}=\mathrm{IRM}_{-0.3 \mathrm{~T}} / \mathrm{IRM}_{1.2 \mathrm{~T}}
$$

\section{Geochemical Analysis}

Samples were analyzed for chemical properties by inductively coupled plasma-atomic emission spectrometry (ICP-AES), inductively coupled plasma-mass spectrometry (ICP-MS) and X-ray fluorescence (XRF) techniques (tables 2 and 5) (Lichte and others, 1987). Sediment and bedrock samples were analyzed on the $<63-\mu \mathrm{m}$ and bulk size fractions, respectively, and were finely ground using a shatterbox.

\section{Acknowledgments}

We thank Yarrow Axford for field assistance; Eric Fisher, Jiang Xiao, Isla Castaneda, Yarrow Axford, and Ken Takagi for sediment analyses; and Darren "Paco” Van Sistine for GIS work. Thanks also to Gary Skipp and Jeff Honke for review of this report and helpful suggestions for improvement.

\section{References Cited}

Bohannon, R.G., 1978, Geologic map of the Las Vegas $1^{\circ} \times 2^{\circ}$ quadrangle, Nevada, Arizona, and California: U.S. Geological Survey Open-File Report 78-670, scale $1: 250,000$.

Dreimanis, Aleksis, 1962, Quantitative gasometric determination of calcite and dolomite by using Chittick apparatus: Journal of Sedimentary Petrology, v. 32, no. 3, p. 520-529.

Eppinger, R.G., Winkler, G.R., Cookro, T.M., Shubat, M.A., Blank, H.R., Crowley, J.K., Kucks, R.P., and Jones, J.L., 1990, Preliminary assessment of the mineral resources of the Cedar City $1^{\circ} \times 2^{\circ}$ quadrangle, Utah: U.S. Geological Survey Open-File Report 90-34, scale 1:250,000.

Goldstein, H.L., Reynolds, R.L., Reheis, M.C., Yount, J.C., and Neff, J.C., in press, Compositional trends in eolian dust along a transect across the southwestern United States: Journal of Geophysical Research — Earth Surface.

Hackman, R.J., and Wyant, D.G., 1973, Geology, structure, and uranium deposits of the Escalante quadrangle, Utah and Arizona: U.S. Geological Survey Miscellaneous Geologic Investigations Map I-744, scale 1:250,000. 
Haynes, D.D., Vogel, J.D., and Wyant, D.G., 1972, Geology, structure, and uranium deposits of the Cortez quadrangle, Colorado and Utah: U.S. Geological Survey Miscellaneous Geologic Investigations Map I-629, scale 1:250,000.

Jennings, C.W., 1961, Geologic map of California, Kingman sheet: State of California, Department of Natural Resources, scale 1:250,000.

King, J.W., and Channel, J.E.T., 1991, Sedimentary magnetism, environmental magnetism, and magnetostratigraphy: Reviews of Geophysics, Supplement, p. 358 - 370.

Litche, F.E., Golightly, D.W., Lamothe, P.J., 1987, Inductively coupled plasma-atomic spectrometry, in Baedecker, P.A., ed., Methods for geochemical analysis: U.S. Geological Survey Bulletin 1770, p. B1-B10.

Machette, M., 1986, Calcium and magnesium carbonates, in Singer, M.J., and Janitzky, P., eds., Field and laboratory procedures used in a soil chronosequence study: U.S. Geological Survey Bulletin 1648, p. 30-33.

Page, W.R., Lundstrom, S.C., Harris, A.G., Langenheim, V.E., Workman, J.B., Mahan, S.A., Paces, J.B., Dixon, G.L., Rowley, P.D., Burchfiel, B.C., Bell, J.W., and Smith, E.I., 2005, Geologic and geophysical maps of the Las Vegas 30’ x 60’ quadrangle, Clark and Nye Counties, Nevada and Inyo County, California: U.S. Geological Survey Scientific Investigations Map 2814, scale 1:100,000.

Singer, M.J., and Janitzky, P., eds., 1986, Field and laboratory procedures used in a soil chronosequence study: U.S. Geological Survey Bulletin 1648, 49 p.

Wenthworth, C.K., 1922, A scale of grade and class terms for clastic sediments: Journal of Geology, v. 30, p. $377-392$.

Williams, P.E., 1964, Geology, structure, and uranium deposits of the Moab quadrangle, Colorado and Utah: U.S. Geological Survey Miscellaneous Geologic Investigation Map I-360, 1:250,000.

Williams, P.E., and Hackman, R.J., 1983, Geology of the Salina quadrangle, Utah: U.S. Geological Survey Miscellaneous Investigations Map I-591-A, 1:250,000. 


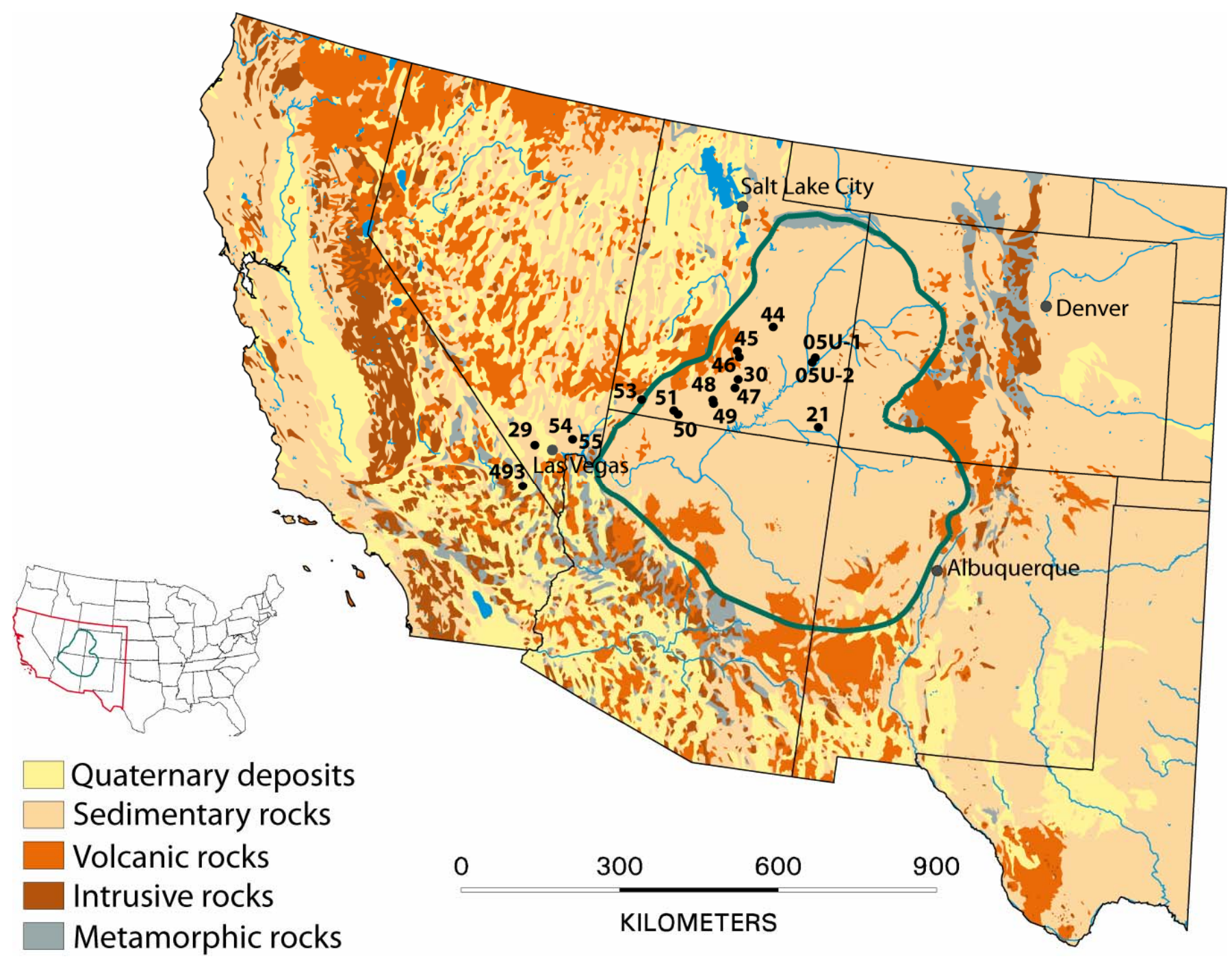

Figure 1. Generalized geologic map of the Southwestern United States showing the southwest-northeast transect of sampled pothole sites (small closed circles). The transect runs from the Mojave Desert to the central Colorado Plateau. Green outline denotes the boundary of the Colorado Plateau. Sites 54 and 55 are in close proximity and are displayed as one point. 
Table 1. Pothole site location information.

\begin{tabular}{|c|c|c|c|c|c|c|}
\hline Sampling site & Latitude $^{1}$ & Longitude $^{1}$ & $\begin{array}{c}\text { Elevation }^{2} \\
\text { (meters) }\end{array}$ & Location & Bedrock type & Geologic map reference \\
\hline 44 & 38.8622 & -110.8369 & 2188 & San Rafael Swell, Utah & Navajo Sandstone & Williams and Hackman, 1983 \\
\hline 45 & 38.3607 & -111.5304 & 2510 & Bicknell, Utah & Navajo Sandstone & Williams and Hackman, 1983 \\
\hline 46 & 38.2601 & -111.4597 & 2262 & Teasdale,Utah & Navajo Sandstone & Williams and Hackman, 1983 \\
\hline 47 & 37.7363 & -111.4414 & 1818 & Escalante, Utah & Navajo Sandstone & Hackman and Wyant, 1973 \\
\hline 48 & 37.4726 & -111.8749 & 2046 & GSENM,Utah ${ }^{3}$ & Entrada Sandstone & Hackman and Wyant, 1973 \\
\hline 49 & 37.4199 & -111.8497 & 1859 & GSENM,Utah ${ }^{3}$ & Navajo Sandstone & Hackman and Wyant, 1973 \\
\hline 50 & 37.1322 & -112.5633 & 1690 & Kanab,Utah & Navajo Sandstone & Eppinger and others, 1990 \\
\hline 51 & 37.1897 & -112.6646 & 1801 & Mt. Carmel Junction,Utah & Carmel Limestone & Eppinger and others, 1990 \\
\hline 53 & 37.2704 & -113.3988 & 1455 & Leeds, Utah & Navajo Sandstone & Eppinger and others, 1990 \\
\hline 54 & 36.3874 & -114.6863 & 906 & Buffington Pocket, Nevada & Aztec Sandstone (redbed) ${ }^{4}$ & Bohannon, 1978 \\
\hline 55 & 36.3867 & -114.6870 & 885 & Buffington Pocket, Nevada & Aztec Sandstone (redbed) ${ }^{4}$ & Bohannon, 1978 \\
\hline 29 & 36.1664 & -115.4522 & 1345 & Red Rocks, Nevada & Aztec Sandstone (redbed) & Page and others, 2005 \\
\hline 30 & 37.8904 & -111.4067 & 1984 & Boulder, Utah & Navajo Sandstone & Hackman and Wyant, 1973 \\
\hline $05 U-1$ & 38.4501 & -109.8169 & 1782 & Island in the Sky, Utah & Navajo Sandstone & Williams, 1964 \\
\hline $05 U-2$ & 38.3668 & -109.8667 & 1879 & Island in the Sky, Utah & Navajo Sandstone & Williams, 1964 \\
\hline 21 & 37.2934 & -109.5354 & 1421 & Bluff, Utah & Bluff Sandstone & Haynes and others, 1972 \\
\hline 493 & 35.4387 & -115.5195 & 1615 & Mescal Range, California & Aztec Sandstone (redbed) ${ }^{4}$ & Jennings, 1961 \\
\hline
\end{tabular}

${ }^{1}$ Latitude and longitude displayed in NAD83 datum.

${ }^{2}$ Elevation determined from 30-m DEM.

${ }^{3}$ GSENM = Grand Staircase Escalante National Monument.

${ }^{4}$ Redbeds contain fine-grained hematite as grain coatings. 


\section{Table 2. Summary data.}

This table lists common soils laboratory data and magnetic and chemical properties completed.

[Sample no., Unique sample number (for example, 44/0-1); presented as site designation (44), and depth in centimeters $(0-1)$.

Hygroscopic moisture factor, A laboratory measure of soil moisture at or near field conditions (driest conditions when values are closest to 1 ).

$\mathrm{CaCO}_{3} \%$, Calcium carbonate percent measured using a Chittick apparatus (Machette, 1986).

Sand, Silt, Clay \%, Sand, silt and clay percentages determined using a laser particle-size analyzer. For detailed particle-size data, see table 3.

Magnetic properties completed, Samples for which magnetic properties were determined. One column lists the magnetic properties that were determined for the $<2$-mm-size fraction and the other column lists the magnetic properties that were determined for the $<63-\mu \mathrm{m}$-size fraction. Refer to text for property designations and appropriate table for specific magnetic property data.

Chemistry completed, Samples for which chemical analysis has been completed. Samples were analyzed by ICPAES, ICP-MS and XRF techniques. Refer to appropriate table for specific chemistry data.

n.d., not determined; mm, millimeter]

\begin{tabular}{|c|c|c|c|c|c|c|c|c|}
\hline $\begin{array}{c}\text { Sample } \\
\text { no. }\end{array}$ & $\begin{array}{l}\text { Hygroscopic } \\
\text { moisture } \\
\text { factor }\end{array}$ & $\begin{array}{c}\mathrm{CaCO}_{3} \\
(\%)\end{array}$ & $\begin{array}{c}\text { Sand } \\
(\%)\end{array}$ & $\begin{array}{l}\text { Silt } \\
(\%)\end{array}$ & $\begin{array}{l}\text { Clay } \\
(\%)\end{array}$ & $\begin{array}{c}\text { Magnetic } \\
\text { properties }(<2 \mathrm{~mm}) \\
\text { completed } \\
\text { (see table 4) }\end{array}$ & $\begin{array}{c}\text { Magnetic } \\
\text { properties (<63 micrometers) } \\
\text { completed } \\
\text { (see table } 4) \\
\end{array}$ & $\begin{array}{c}\text { Chemistry completed } \\
\text { (see table 5) }\end{array}$ \\
\hline $44 / 0-1$ & 0.997 & 1.07 & 75.98 & 18.48 & 3.89 & MS, ARM, IRM & MS, ARM, IRM & ICP-AES, ICP-MS, XRF \\
\hline $44 / 1-2$ & 0.996 & 0.88 & 79.35 & 13.98 & 5.07 & MS, ARM, IRM & MS, ARM, IRM & ICP-AES, ICP-MS, XRF \\
\hline $44 / 2-5$ & 0.996 & 0.58 & 80.30 & 11.53 & 6.30 & MS, ARM, IRM & MS, ARM, IRM & ICP-AES, ICP-MS, XRF \\
\hline 44/Bedrock & n.d. & n.d. & 94.73 & 3.11 & 2.16 & MS, ARM, IRM & n.d. & ICP-AES, ICP-MS, XRF \\
\hline $45 / 0-1$ & 0.998 & 0.17 & 86.88 & 9.21 & 3.21 & MS, ARM, IRM & MS, ARM, IRM & ICP-AES, ICP-MS, XRF \\
\hline $45 / 1-2$ & 0.998 & 0.26 & 86.53 & 8.20 & 4.42 & MS, ARM, IRM & MS, ARM, IRM & ICP-AES, ICP-MS, XRF \\
\hline $45 / 2-5$ & 0.997 & 0.31 & 83.10 & 9.57 & 6.04 & MS, ARM, IRM & MS, ARM, IRM & ICP-AES, ICP-MS, XRF \\
\hline 45/Bedrock & n.d. & n.d. & 91.35 & 5.62 & 3.03 & MS, ARM, IRM & n.d. & ICP-AES, ICP-MS, XRF \\
\hline $46 / 0-1$ & 0.997 & 0.22 & 92.30 & 4.33 & 2.70 & MS, ARM, IRM & MS, ARM, IRM & n.d. \\
\hline $46 / 1-2$ & 0.997 & 0.27 & 88.37 & 6.10 & 4.53 & MS, ARM, IRM & MS, ARM, IRM & n.d. \\
\hline $46 / 2-5$ & 0.997 & 0.21 & 76.07 & 13.93 & 7.95 & MS, ARM, IRM & MS, ARM, IRM & n.d. \\
\hline 46/Bedrock & n.d. & n.d. & n.d. & n.d. & n.d. & n.d. & n.d. & n.d. \\
\hline $47 / 0-1$ & 0.997 & 0.24 & 37.00 & 44.07 & 13.01 & MS, ARM, IRM & MS, ARM, IRM & ICP-AES, ICP-MS, XRF \\
\hline $47 / 1-2$ & 0.997 & 0.23 & 29.80 & 46.36 & 17.77 & MS, ARM, IRM & MS, ARM, IRM & ICP-AES, ICP-MS, XRF \\
\hline $47 / 2-5$ & 0.998 & 0.28 & 23.95 & 43.48 & 24.01 & MS, ARM, IRM & MS, ARM, IRM & ICP-AES, ICP-MS, XRF \\
\hline 47/Bedrock & n.d. & n.d. & 96.50 & 2.54 & 0.96 & MS, ARM, IRM & n.d. & ICP-AES, ICP-MS, XRF \\
\hline $48 / 0-1$ & 0.997 & 4.65 & 71.53 & 16.14 & 9.17 & MS, ARM, IRM & MS, ARM, IRM & ICP-AES, ICP-MS, XRF \\
\hline $48 / 1-2$ & 0.997 & 4.97 & 76.13 & 11.72 & 8.95 & MS, ARM, IRM & MS, ARM, IRM & ICP-AES, ICP-MS, XRF \\
\hline $48 / 2-5$ & 0.997 & 5.51 & 74.02 & 11.93 & 9.57 & MS, ARM, IRM & MS, ARM, IRM & ICP-AES, ICP-MS, XRF \\
\hline 48/Bedrock & n.d. & n.d. & 88.80 & 7.13 & 4.08 & MS, ARM, IRM & n.d. & ICP-AES, ICP-MS, XRF \\
\hline $49 / 0-1$ & 0.998 & 0.37 & 80.93 & 14.14 & 3.47 & MS, ARM, IRM & MS, ARM, IRM & ICP-AES, ICP-MS, XRF \\
\hline $49 / 1-2$ & 0.998 & 0.36 & 84.67 & 10.17 & 3.58 & MS, ARM, IRM & MS, ARM, IRM & ICP-AES, ICP-MS, XRF \\
\hline $49 / 2-5$ & 0.997 & 0.31 & 83.97 & 10.30 & 4.47 & MS, ARM, IRM & n.d. & n.d. \\
\hline 49/Bedrock & n.d. & n.d. & 97.59 & 1.44 & 0.97 & MS, ARM, IRM & n.d. & ICP-AES, ICP-MS \\
\hline
\end{tabular}


Table 2. Summary data.-Continued

\begin{tabular}{|c|c|c|c|c|c|c|c|c|}
\hline $\begin{array}{c}\text { Sample } \\
\text { no. }\end{array}$ & $\begin{array}{l}\text { Hygroscopic } \\
\text { moisture } \\
\text { factor }\end{array}$ & $\begin{array}{c}\mathrm{CaCO}_{3} \\
(\%)\end{array}$ & $\begin{array}{c}\text { Sand } \\
(\%)\end{array}$ & $\begin{array}{l}\text { Silt } \\
(\%)\end{array}$ & $\begin{array}{l}\text { Clay } \\
(\%)\end{array}$ & $\begin{array}{c}\text { Magnetic } \\
\text { properties }(<2 \mathrm{~mm}) \\
\text { completed } \\
(\text { see table 4) } \\
\end{array}$ & $\begin{array}{c}\text { Magnetic } \\
\text { properties (<63 micrometers) } \\
\text { completed } \\
\text { (see table 4) } \\
\end{array}$ & $\begin{array}{c}\text { Chemistry completed } \\
\text { (see table 5) }\end{array}$ \\
\hline $50 / 0-1$ & 0.997 & 0.76 & 90.38 & 7.69 & 1.40 & MS, ARM, IRM & MS, ARM, IRM & ICP-AES, ICP-MS, XRF \\
\hline $50 / 1-2$ & 0.997 & 0.93 & 83.88 & 13.08 & 2.19 & MS, ARM, IRM & MS, ARM, IRM & ICP-AES, ICP-MS, XRF \\
\hline $50 / 2-5$ & 0.998 & 1.04 & 80.64 & 15.71 & 2.41 & MS, ARM, IRM & MS, ARM, IRM & ICP-AES, ICP-MS, XRF \\
\hline 50/Bedrock & n.d. & n.d. & 100.00 & 0.00 & 0.00 & MS, ARM, IRM & n.d. & ICP-AES, ICP-MS, XRF \\
\hline $51 / 0-1$ & 0.998 & 0.20 & 77.06 & 17.08 & 4.18 & MS, ARM, IRM & MS, ARM, IRM & ICP-AES, ICP-MS, XRF \\
\hline $51 / 1-2$ & 0.996 & 0.22 & 81.38 & 12.59 & 4.37 & MS, ARM, IRM & MS, ARM, IRM & ICP-AES, ICP-MS, XRF \\
\hline $51 / 2-5$ & 0.998 & 0.38 & 82.42 & 10.27 & 5.15 & MS, ARM, IRM & MS, ARM, IRM & ICP-AES, ICP-MS, XRF \\
\hline 51/Bedrock & n.d. & n.d. & n.d. & n.d. & n.d. & MS, ARM, IRM & n.d. & ICP-AES, ICP-MS, XRF \\
\hline $53 / 0-1$ & 0.997 & 0.14 & 78.98 & 16.54 & 3.58 & MS, ARM, IRM & MS, ARM, IRM & ICP-AES, ICP-MS, XRF \\
\hline $53 / 1-2$ & 0.997 & 0.13 & 85.82 & 9.69 & 3.47 & MS, ARM, IRM & MS, ARM, IRM & ICP-AES, ICP-MS, XRF \\
\hline $53 / 2-5$ & 0.998 & 0.13 & 43.82 & 28.32 & 20.81 & MS, ARM, IRM & MS, ARM, IRM & ICP-AES, ICP-MS, XRF \\
\hline 53/Bedrock & n.d. & n.d. & 93.32 & 4.94 & 1.74 & MS, ARM, IRM & n.d. & ICP-AES, ICP-MS, XRF \\
\hline $54 / 0-1$ & 0.998 & 0.18 & 65.17 & 27.83 & 4.93 & MS, ARM, IRM & MS, ARM, IRM & ICP-AES, ICP-MS, XRF \\
\hline $54 / 1-2$ & 0.998 & 0.16 & 68.74 & 17.07 & 9.79 & MS, ARM, IRM & MS, ARM, IRM & ICP-AES, ICP-MS, XRF \\
\hline $54 / 2-5$ & 0.998 & 0.13 & 63.41 & 16.73 & 13.08 & MS, ARM, IRM & MS, ARM, IRM & ICP-AES, ICP-MS, XRF \\
\hline 54/Bedrock & n.d. & n.d. & 77.09 & 3.22 & 19.26 & MS, ARM, IRM & n.d. & ICP-AES, ICP-MS, XRF \\
\hline $55 / 0-1$ & 0.996 & 0.75 & 66.51 & 26.18 & 5.10 & MS, ARM, IRM & MS, ARM, IRM & ICP-AES, ICP-MS, XRF \\
\hline $55 / 1-2$ & 0.998 & 0.34 & 59.78 & 26.20 & 9.47 & MS, ARM, IRM & MS, ARM, IRM & ICP-AES, ICP-MS, XRF \\
\hline $55 / 2-5$ & 0.997 & 0.23 & 64.72 & 21.46 & 8.97 & MS, ARM, IRM & MS, ARM, IRM & ICP-AES, ICP-MS, XRF \\
\hline 55/Bedrock & n.d. & n.d. & 95.71 & 2.49 & 1.80 & MS, ARM, IRM & n.d. & ICP-AES, ICP-MS, XRF \\
\hline $29 / 0-1$ & 0.999 & 0.19 & 68.35 & 23.49 & 5.19 & MS, ARM, IRM & MS, ARM, IRM & ICP-AES, ICP-MS, XRF \\
\hline $29 / 1-2$ & 0.998 & 0.23 & 56.98 & 27.62 & 10.87 & MS, ARM, IRM & MS, ARM, IRM & ICP-AES, ICP-MS, XRF \\
\hline $29 / 2-5$ & 0.997 & 0.20 & 53.68 & 28.34 & 13.59 & MS, ARM, IRM & MS, ARM, IRM & ICP-AES, ICP-MS, XRF \\
\hline 29/Bedrock & n.d. & n.d. & 92.28 & 5.42 & 2.30 & MS, ARM, IRM & n.d. & ICP-AES, ICP-MS, XRF \\
\hline $30 / 0-1$ & 0.997 & 0.26 & 66.97 & 23.17 & 7.98 & MS, ARM, IRM & MS, ARM, IRM & ICP-AES, ICP-MS, XRF \\
\hline $30 / 1-2$ & 0.998 & 0.10 & 44.67 & 31.34 & 17.49 & MS, ARM, IRM & MS, ARM, IRM & ICP-AES, ICP-MS, XRF \\
\hline $30 / 2-5$ & 0.997 & 0.21 & 46.06 & 22.63 & 20.92 & MS, ARM, IRM & MS, ARM, IRM & ICP-AES, ICP-MS, XRF \\
\hline 30/Bedrock & n.d. & n.d. & 91.97 & 5.50 & 2.53 & MS, ARM, IRM & n.d. & ICP-AES, ICP-MS, XRF \\
\hline $05 U-1 / 0-1$ & 0.994 & 1.02 & 80.61 & 13.13 & 5.04 & MS, ARM, IRM & MS, ARM, IRM & ICP-AES, ICP-MS, XRF \\
\hline $05 U-1 / 1-2$ & 0.991 & 0.22 & 89.77 & 5.74 & 3.64 & MS, ARM, IRM & MS, ARM, IRM & ICP-AES, ICP-MS, XRF \\
\hline 05U-1/Bedrock & n.d. & 1.21 & 95.88 & 2.40 & 1.72 & MS, ARM, IRM & n.d. & ICP-AES, ICP-MS, XRF \\
\hline $05 \mathrm{U}-2 / 0-1$ & 0.996 & 0.36 & 74.65 & 17.82 & 6.10 & MS, ARM, IRM & MS, ARM, IRM & ICP-AES, ICP-MS, XRF \\
\hline $05 U-2 / 1-2$ & 0.993 & 0.27 & 76.56 & 15.85 & 6.15 & MS, ARM, IRM & MS, ARM, IRM & ICP-AES, ICP-MS, XRF \\
\hline $05 U-2 / 2-5$ & 0.950 & 0.24 & 82.96 & 10.96 & 4.78 & MS, ARM, IRM & MS, ARM, IRM & ICP-AES, ICP-MS, XRF \\
\hline 05U-2/Bedrock & n.d. & 0.19 & 96.27 & 2.01 & 1.73 & MS, ARM, IRM & n.d. & ICP-AES, ICP-MS, XRF \\
\hline $21 / 0-1$ & 0.990 & 2.25 & 94.11 & 3.56 & 1.93 & MS, ARM, IRM & MS, ARM, IRM & ICP-AES, ICP-MS, XRF \\
\hline $21 / 1-2$ & 0.960 & 1.71 & 92.95 & 4.34 & 2.24 & MS, ARM, IRM & MS, ARM, IRM & ICP-AES, ICP-MS, XRF \\
\hline $21 / 2-5$ & 0.942 & 1.68 & 92.83 & 4.57 & 2.07 & MS, ARM, IRM & MS, ARM, IRM & ICP-AES, ICP-MS, XRF \\
\hline 21/Bedrock & n.d. & 2.15 & 94.45 & 3.11 & 2.44 & MS, ARM, IRM & n.d. & ICP-AES, ICP-MS, XRF \\
\hline $493 / 0-1$ & 0.992 & 0.28 & 62.65 & 31.23 & 3.93 & MS, ARM, IRM & MS, ARM, IRM & ICP-AES, ICP-MS, XRF \\
\hline $493 / 1-2$ & 0.991 & 0.20 & 59.96 & 32.39 & 5.83 & MS, ARM, IRM & MS, ARM, IRM & ICP-AES, ICP-MS, XRF \\
\hline $493 / 2-5$ & 0.995 & 0.32 & 72.48 & 21.64 & 4.24 & MS, ARM, IRM & MS, ARM, IRM & ICP-AES, ICP-MS, XRF \\
\hline 493/Bedrock & n.d. & n.d. & n.d. & n.d. & n.d. & n.d. & n.d. & ICP-AES, ICP-MS, XRF \\
\hline
\end{tabular}


Table 3. Particle size data.

This table contains high-resolution particle-size data obtained using a laser particle-size analyzer. The particle size is displayed in accordance with the Wentworth scale (Wentworth, 1922). Values are reported as volume percent. Each value corresponds to the volume percent of the sample that has a grain size falling within that range.

[microns, micrometer; n.d., not determined; cm, centimeter]

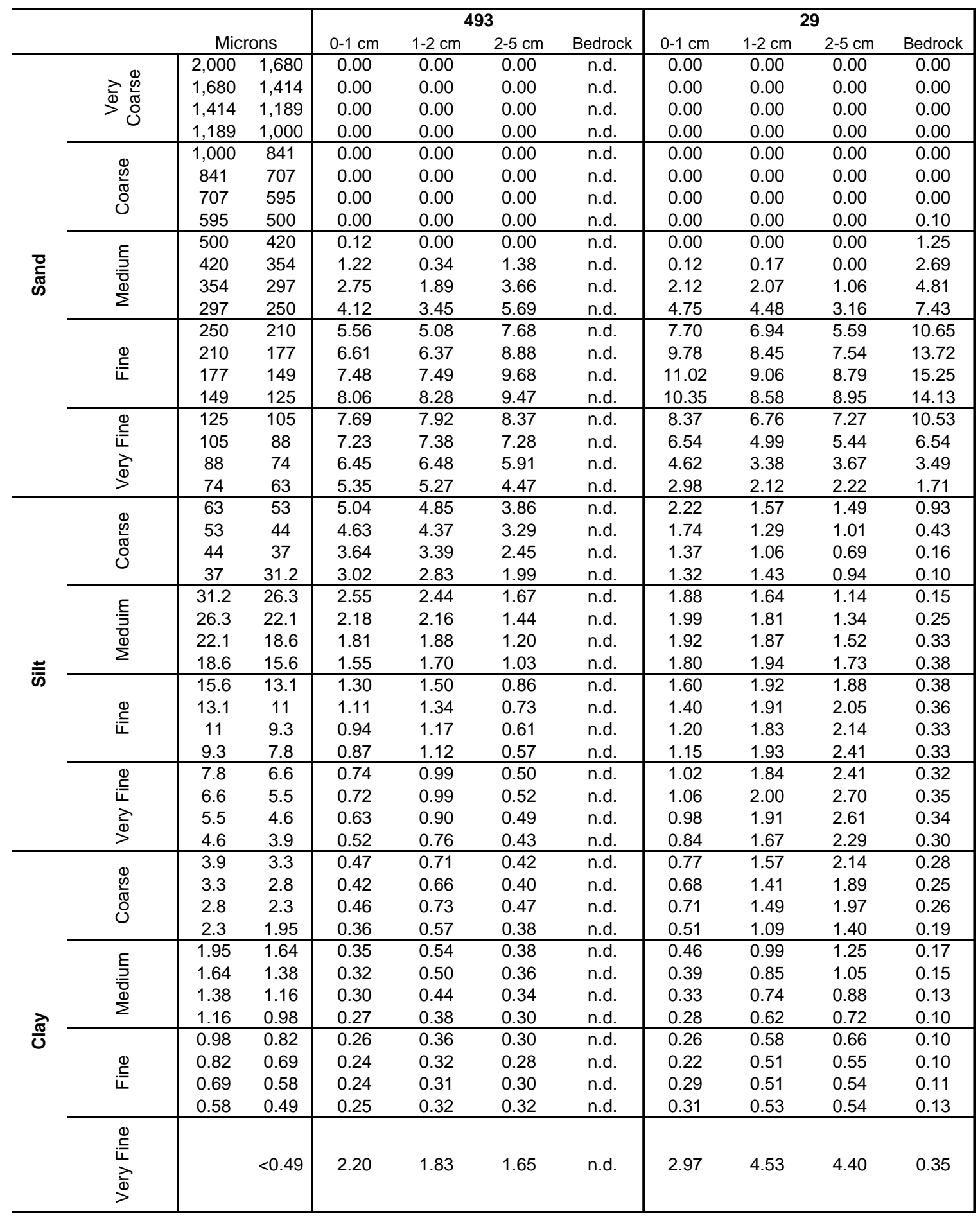


Table 3. Particle size data.-Continued

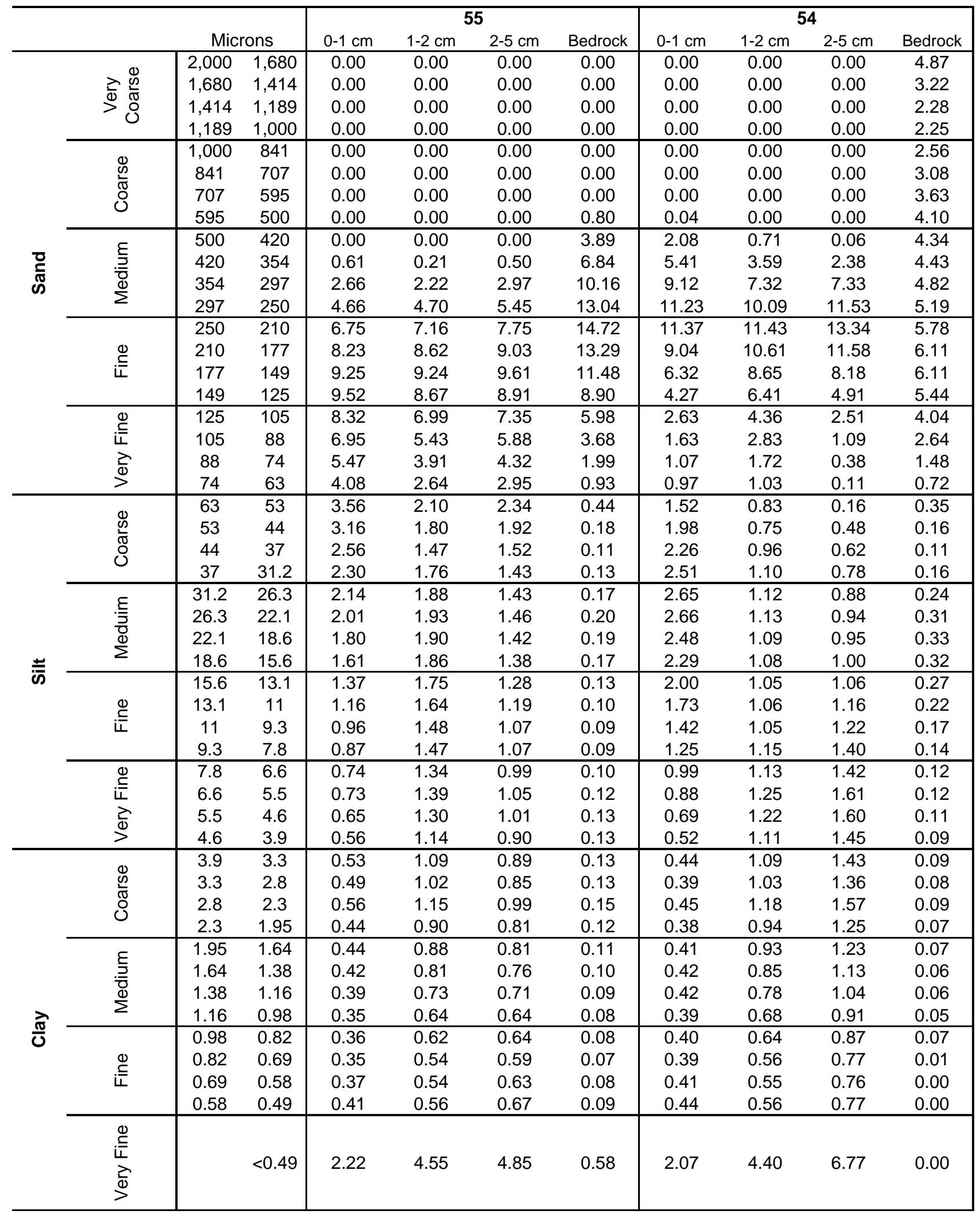


Table 3. Particle size data.-Continued

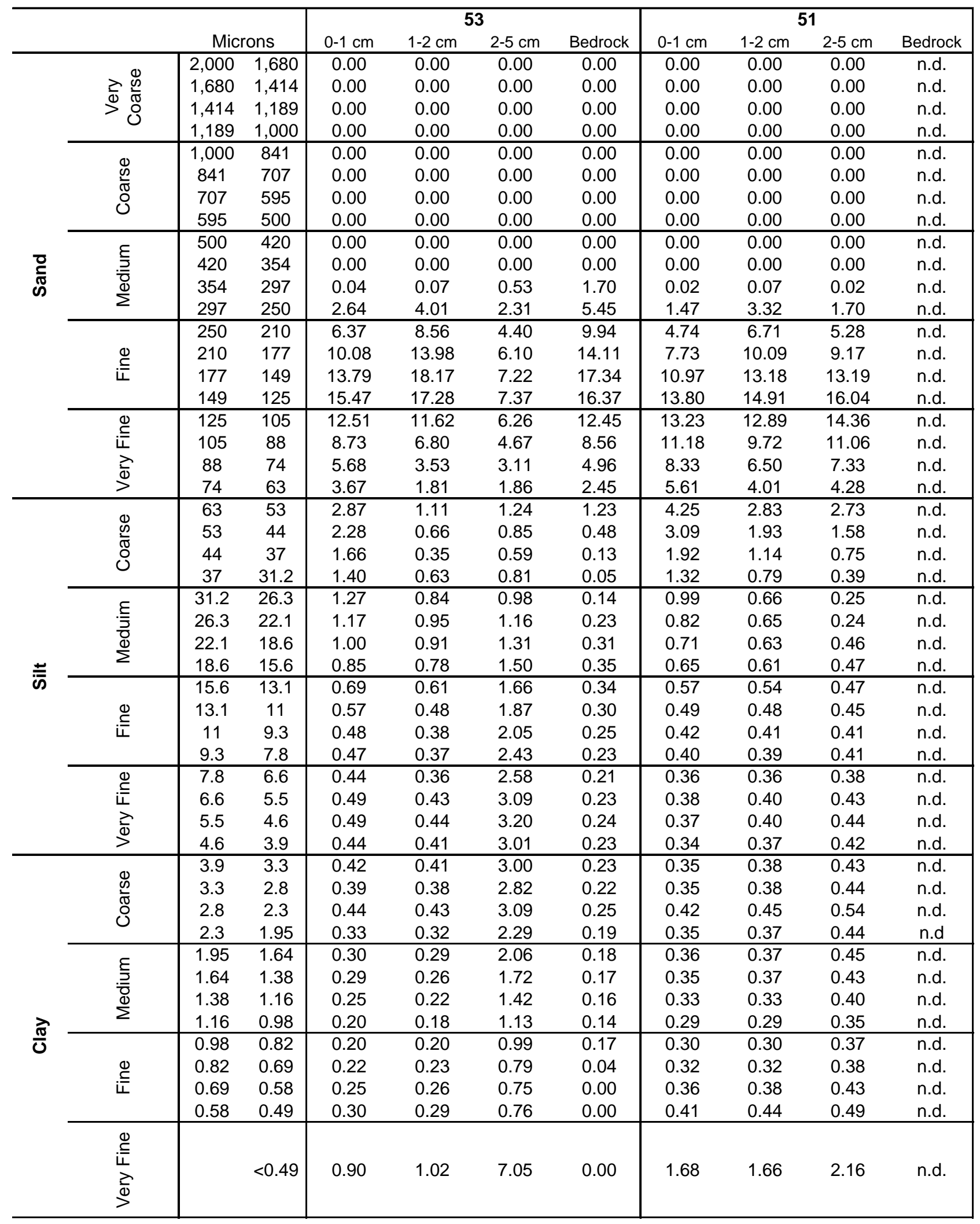


Table 3. Particle size data.-Continued

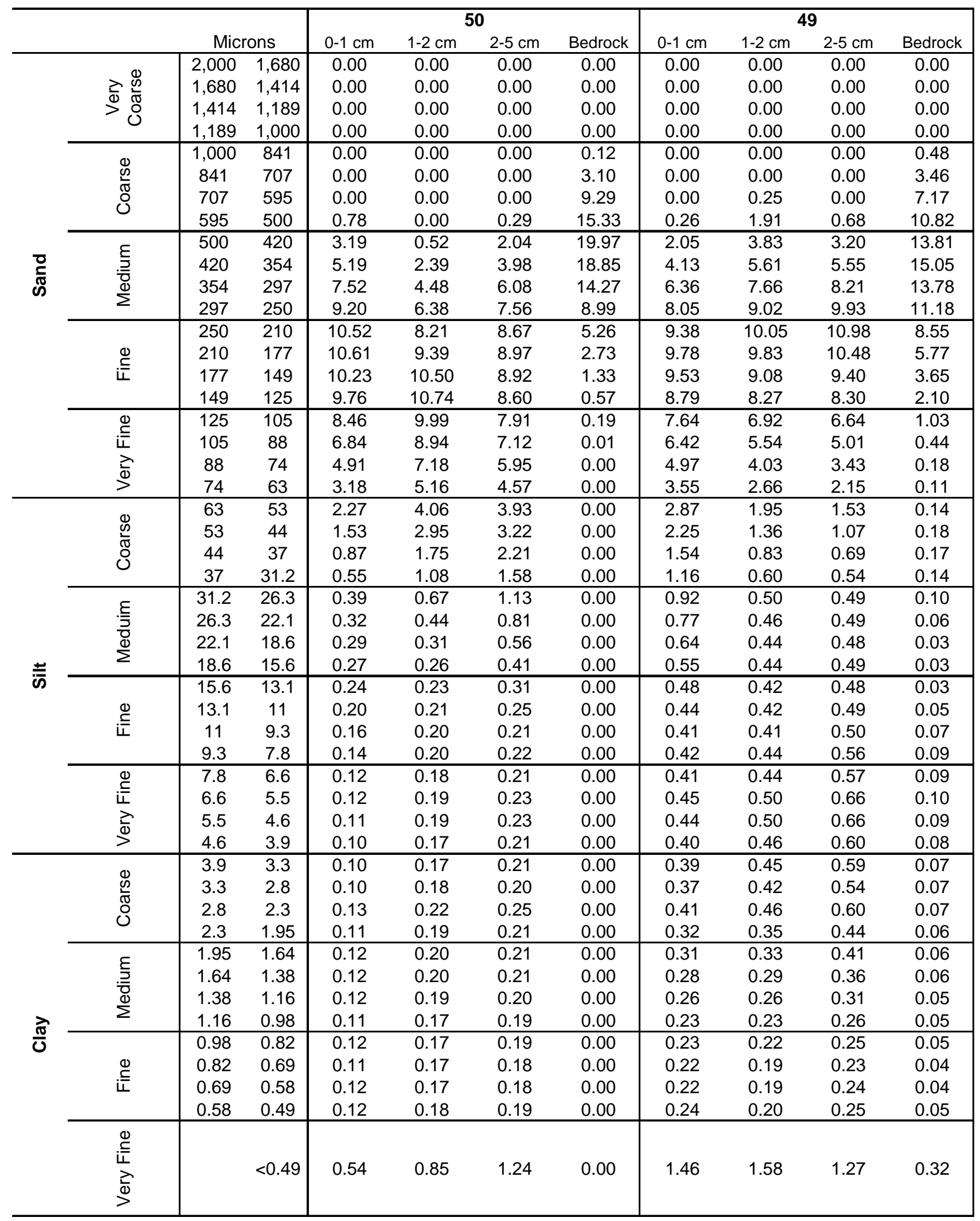


Table 3. Particle size data.-Continued

\begin{tabular}{|c|c|c|c|c|c|c|c|c|c|c|c|}
\hline & & & & & & & & & & & \\
\hline & & Mic & ons & $0-1 \mathrm{~cm}$ & $1-2 \mathrm{~cm}$ & $2-5 \mathrm{~cm}$ & Bedrock & $0-1 \mathrm{~cm}$ & $1-2 \mathrm{~cm}$ & $2-5 \mathrm{~cm}$ & Bedrock \\
\hline & & 2,000 & 1,680 & 0.00 & 0.00 & 0.00 & 0.00 & 0.00 & 0.00 & 0.00 & 0.00 \\
\hline & $\geq \underset{0}{\infty}$ & 1,680 & 1,414 & 0.00 & 0.00 & 0.00 & 0.00 & 0.00 & 0.00 & 0.00 & 0.00 \\
\hline & $>0$ & 1,414 & 1,189 & 0.00 & 0.00 & 0.00 & 0.00 & 0.00 & 0.00 & 0.00 & 0.00 \\
\hline & & 1,189 & 1,000 & 0.00 & 0.00 & 0.00 & 0.00 & 0.00 & 0.00 & 0.00 & 0.00 \\
\hline & & 1,000 & 841 & 0.00 & 0.00 & 0.00 & 0.00 & 0.00 & 0.00 & 0.00 & 0.00 \\
\hline & $\stackrel{\frac{\omega}{\sigma}}{\bar{\sigma}}$ & 841 & 707 & 0.00 & 0.00 & 0.00 & 0.01 & 0.00 & 0.00 & 0.00 & 0.00 \\
\hline & 8 & 707 & 595 & 0.00 & 0.00 & 0.00 & 1.35 & 0.00 & 0.00 & 0.00 & 0.52 \\
\hline & & 595 & 500 & 0.00 & 0.00 & 0.00 & 4.19 & 0.00 & 0.00 & 0.00 & 3.64 \\
\hline & & 500 & 420 & 0.00 & 0.00 & 0.00 & 6.82 & 0.00 & 0.00 & 0.00 & 7.31 \\
\hline 兽 & s. & 420 & 354 & 0.00 & 0.00 & 0.00 & 9.32 & 0.00 & 0.43 & 0.00 & 11.54 \\
\hline ஸ் & $\stackrel{8}{2}$ & 354 & 297 & 0.03 & 0.03 & 0.00 & 11.54 & 2.08 & 2.32 & 1.48 & 15.76 \\
\hline & & 297 & 250 & 1.70 & 1.78 & 0.44 & 11.92 & 3.68 & 4.13 & 2.74 & 16.94 \\
\hline & & 250 & 210 & 3.93 & 4.41 & 2.60 & 10.73 & 5.25 & 5.22 & 3.97 & 14.42 \\
\hline & $\stackrel{Ð}{=}$ & 210 & 177 & 6.21 & 7.25 & 5.42 & 9.08 & 5.63 & 5.14 & 4.26 & 10.99 \\
\hline & 泣 & 177 & 149 & 9.00 & 10.39 & 8.99 & 7.45 & 5.35 & 4.39 & 3.95 & 7.49 \\
\hline & & 149 & 125 & 11.75 & 13.58 & 12.90 & 5.84 & 4.52 & 3.17 & 3.13 & 4.43 \\
\hline & $\Phi$ & 125 & 105 & 12.99 & 13.49 & 15.20 & 4.21 & 3.58 & 2.14 & 1.99 & 2.17 \\
\hline & 谇 & 105 & 88 & 11.47 & 11.64 & 13.26 & 2.98 & 2.85 & 1.38 & 1.30 & 0.91 \\
\hline & $\frac{\pi}{0}$ & 88 & 74 & 8.69 & 8.42 & 9.52 & 2.01 & 2.24 & 0.85 & 0.74 & 0.31 \\
\hline & 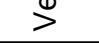 & 74 & 63 & 5.76 & 5.13 & 5.67 & 1.34 & 1.83 & 0.63 & 0.40 & 0.08 \\
\hline & & 63 & 53 & 4.13 & 3.27 & 3.43 & 1.08 & 1.94 & 0.77 & 0.34 & 0.02 \\
\hline & $\stackrel{\omega}{\sigma}$ & 53 & 44 & 2.71 & 1.84 & 1.72 & 0.91 & 2.27 & 1.17 & 0.48 & 0.05 \\
\hline & రొ & 44 & 37 & 1.46 & 0.80 & 0.63 & 0.69 & 2.39 & 1.51 & 0.70 & 0.11 \\
\hline & & 37 & 31.2 & 0.82 & 0.34 & 0.21 & 0.57 & 2.62 & 1.89 & 0.99 & 0.15 \\
\hline & & 31.2 & 26.3 & 0.50 & 0.16 & 0.07 & 0.47 & 2.84 & 2.25 & 1.32 & 0.18 \\
\hline & 堃 & 26.3 & 22.1 & 0.40 & 0.12 & 0.05 & 0.40 & 3.02 & 2.59 & 1.70 & 0.19 \\
\hline & d & 22.1 & 18.6 & 0.43 & 0.28 & 0.27 & 0.33 & 3.05 & 2.83 & 2.06 & 0.17 \\
\hline \pm & & 18.6 & 15.6 & 0.51 & 0.36 & 0.39 & 0.29 & 3.12 & 3.13 & 2.52 & 0.15 \\
\hline "் & & 15.6 & 13.1 & 0.56 & 0.43 & 0.50 & 0.27 & 3.08 & 3.36 & 2.98 & 0.14 \\
\hline & 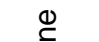 & 13.1 & 11 & 0.59 & 0.48 & 0.57 & 0.27 & 3.07 & 3.62 & 3.49 & 0.15 \\
\hline & i亡 & 11 & 9.3 & 0.58 & 0.48 & 0.58 & 0.27 & 2.93 & 3.70 & 3.83 & 0.16 \\
\hline & & 9.3 & 7.8 & 0.62 & 0.53 & 0.64 & 0.30 & 3.04 & 4.07 & 4.45 & 0.20 \\
\hline & $\Phi$ & 7.8 & 6.6 & 0.61 & 0.55 & 0.63 & 0.30 & 2.83 & 3.94 & 4.52 & 0.22 \\
\hline & 泣 & 6.6 & 5.5 & 0.72 & 0.66 & 0.73 & 0.34 & 2.95 & 4.25 & 5.05 & 0.25 \\
\hline & $\geq$ & 5.5 & 4.6 & 0.76 & 0.71 & 0.77 & 0.33 & 2.68 & 3.95 & 4.84 & 0.23 \\
\hline & & 4.6 & 3.9 & 0.75 & 0.72 & 0.75 & 0.30 & 2.24 & 3.34 & 4.20 & 0.19 \\
\hline & & 3.9 & 3.3 & 0.80 & 0.78 & 0.81 & 0.29 & 2.01 & 3.01 & 3.87 & 0.16 \\
\hline & $\stackrel{\omega}{\omega}$ & 3.3 & 2.8 & 0.82 & 0.80 & 0.83 & 0.28 & 1.73 & 2.57 & 3.38 & 0.13 \\
\hline & ర్ & 2.8 & 2.3 & 1.00 & 0.98 & 1.01 & 0.31 & 1.76 & 2.59 & 3.46 & 0.13 \\
\hline & & 2.3 & 1.95 & 0.82 & 0.81 & 0.84 & 0.25 & 1.25 & 1.79 & 2.43 & 0.09 \\
\hline & & 1.95 & 1.64 & 0.81 & 0.80 & 0.85 & 0.24 & 1.11 & 1.55 & 2.14 & 0.09 \\
\hline & 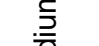 & 1.64 & 1.38 & 0.77 & 0.76 & 0.80 & 0.22 & 0.95 & 1.28 & 1.78 & 0.08 \\
\hline & 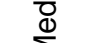 & 1.38 & 1.16 & 0.70 & 0.69 & 0.75 & 0.20 & 0.83 & 1.08 & 1.50 & 0.08 \\
\hline & & 1.16 & 0.98 & 0.62 & 0.62 & 0.69 & 0.18 & 0.71 & 0.89 & 1.25 & 0.08 \\
\hline$\frac{\pi}{0}$ & & 0.98 & 0.82 & 0.65 & 0.64 & 0.71 & 0.17 & 0.69 & 0.83 & 1.16 & 0.10 \\
\hline & $\stackrel{\oplus}{=}$ & 0.82 & 0.69 & 0.66 & 0.64 & 0.70 & 0.16 & 0.63 & 0.72 & 1.01 & 0.02 \\
\hline & i亡 & 0.69 & 0.58 & 0.73 & 0.69 & 0.76 & 0.18 & 0.65 & 0.72 & 1.01 & 0.00 \\
\hline & & 0.58 & 0.49 & 0.80 & 0.74 & 0.82 & 0.20 & 0.69 & 0.75 & 1.04 & 0.00 \\
\hline & $\frac{\stackrel{Q}{\frac{D}{4}}}{\stackrel{\frac{1}{0}}{>}}$ & & $<0.49$ & 3.16 & 3.20 & 4.49 & 1.41 & 5.93 & 6.06 & 8.56 & 0.00 \\
\hline
\end{tabular}


Table 3. Particle size data.-Continued

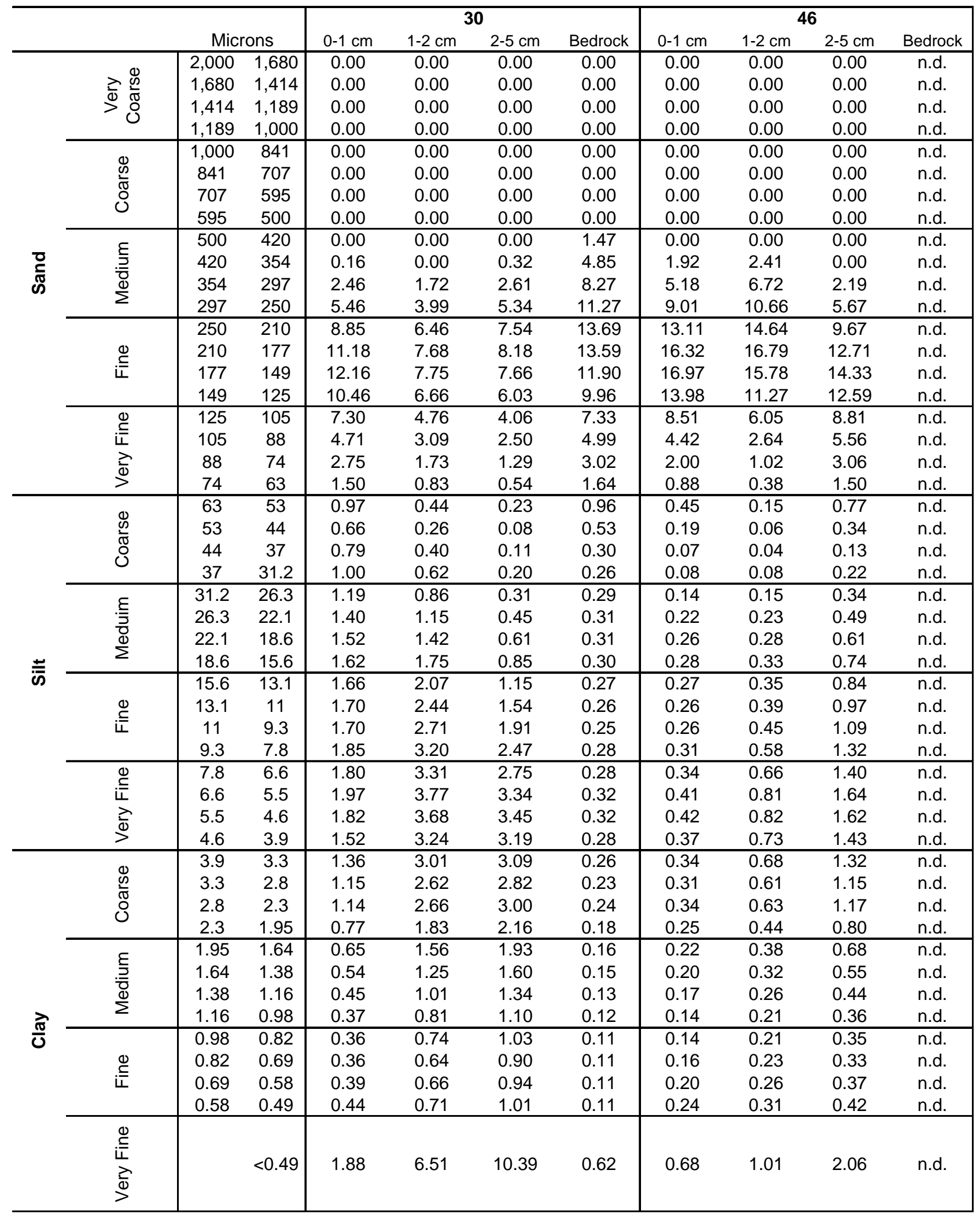


Table 3. Particle size data.-Continued

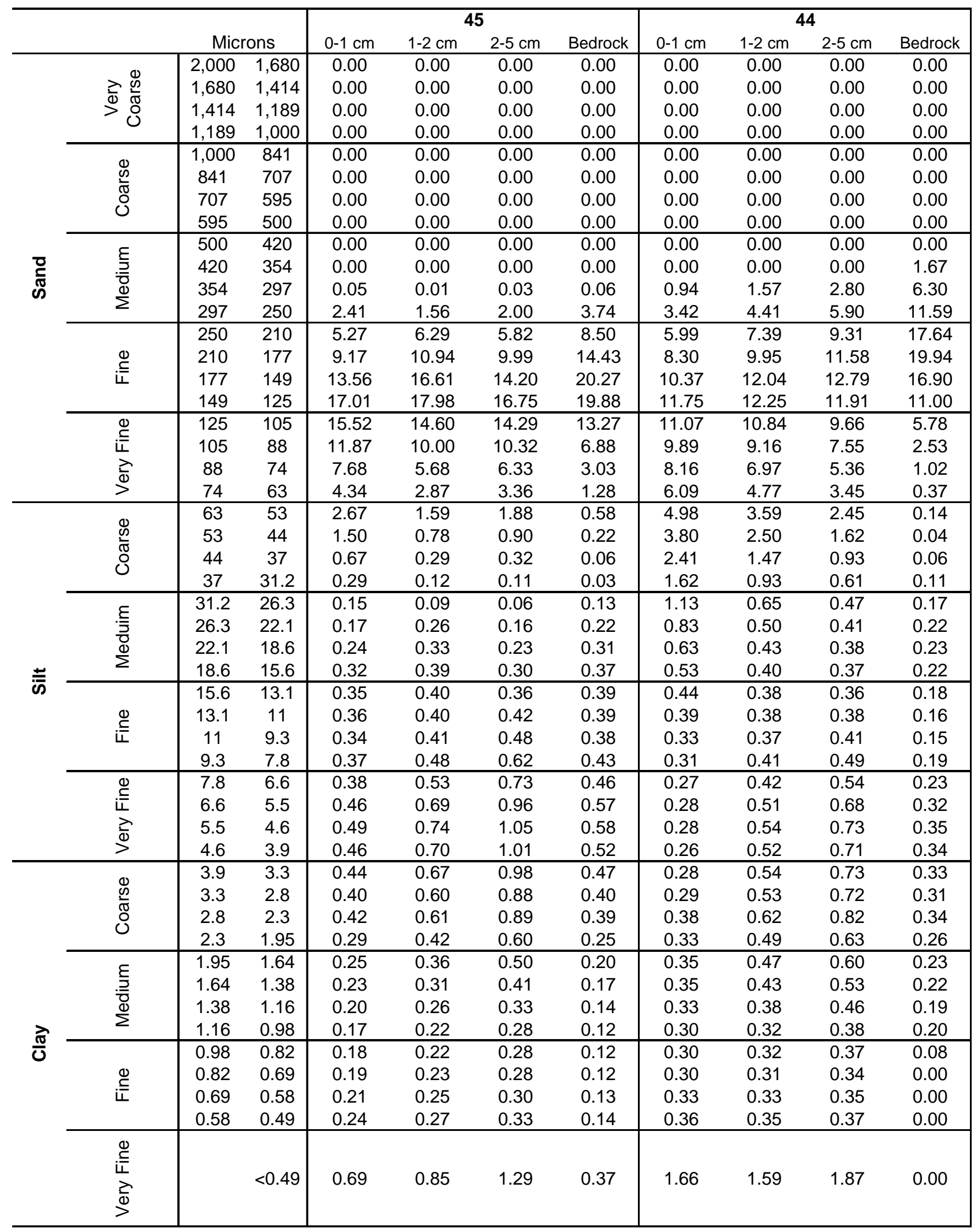


Table 3. Particle size data.-Continued

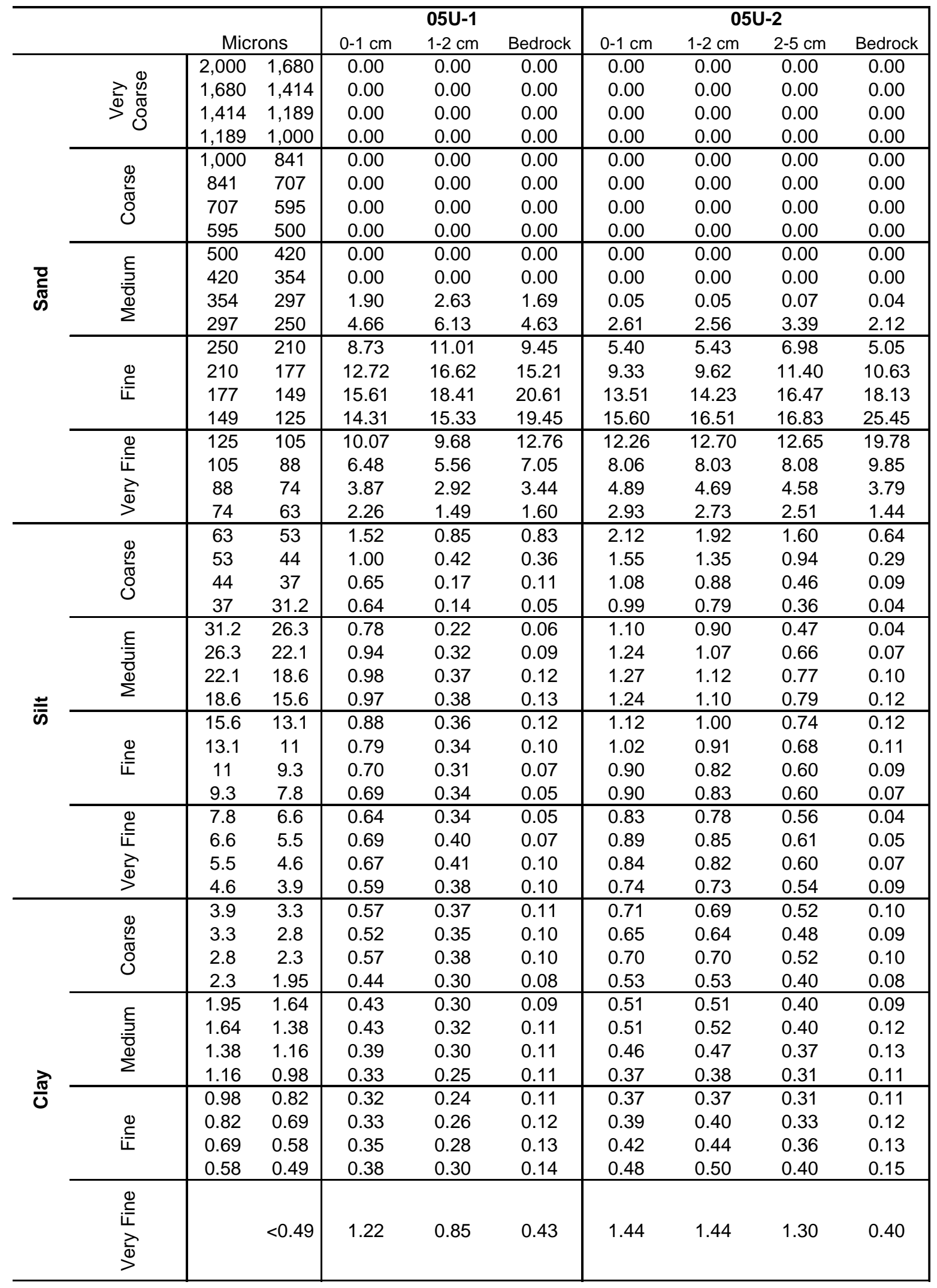


Table 3. Particle size data.-Continued

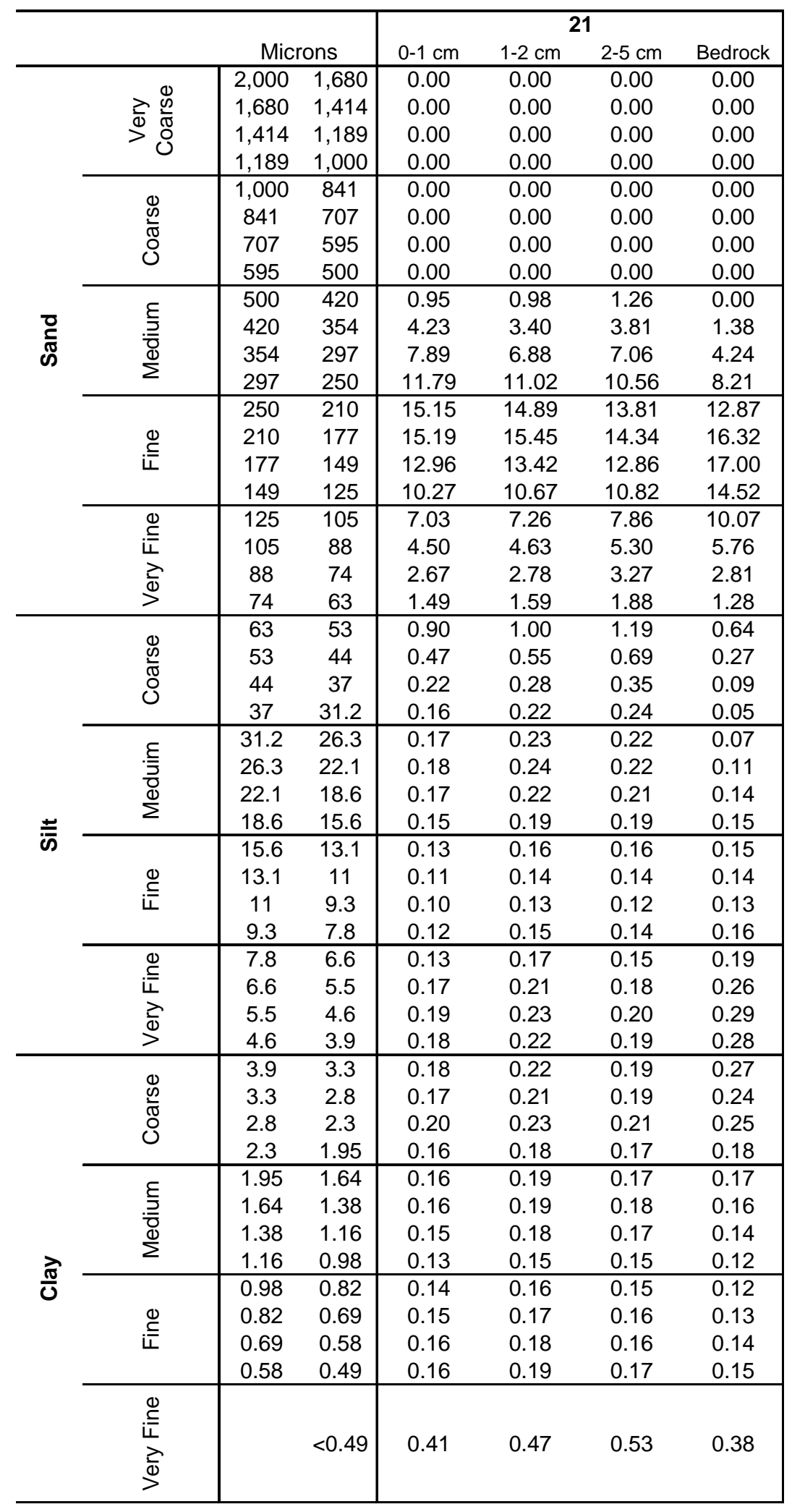




\section{Table 4. Magnetic properties.}

[Sample no., Unique sample number (for example, 44/0-1); presented as site designation (44), and depth in centimeters $(0-1)$.

MSlf and MShf, Low frequency (lf) and high frequency (hf) magnetic susceptibility in $\mathrm{m}^{3} / \mathrm{kg}$.

FDMS, Frequency-dependent magnetic susceptibility. Expressed in percent and $\mathrm{m}^{3} / \mathrm{kg}$.

ARM, Anhysteretic remanent magnetization in $\mathrm{Am}^{2} / \mathrm{kg}$.

IRM 1.2, Isothermal remanent magnetization from a 1.2 tesla induction. Expressed in $\mathrm{Am}^{2} / \mathrm{kg}$.

IRM -0.3, Isothermal remanent magnetization from a -0.3 tesla induction. Expressed in $\mathrm{Am}^{2} / \mathrm{kg}$.

HIRM, Hard isothermal remanent magnetization: HIRM is calculated as: (IRM1.2T + IRM-0.3T)/2.

S, (S parameter) calculated as IRM-0.3T/IRM1.2T.

n.d., not determined; $\mathbf{A m}^{2} / \mathbf{k g}$, Ampere meter squared per kilogram; $\mathbf{m}^{3} / \mathbf{k g}$, cubic meter per kilogram; mm, millimeter; $\mathbf{T}$, tesla; $\boldsymbol{\mu m}$, micrometer; $\%$, percent]

Magnetic properties for $<2$-mm-size fraction and bulk bedrock.

\begin{tabular}{|c|c|c|c|c|c|c|c|c|c|}
\hline Sample no. & $\begin{array}{c}\text { MSIf } \\
\left(\mathrm{m}^{3} / \mathrm{kg}\right)\end{array}$ & $\begin{array}{c}\text { MShf } \\
\left(\mathrm{m}^{3} / \mathrm{kg}\right)\end{array}$ & $\begin{array}{c}\text { FDMS } \\
(\%)\end{array}$ & $\begin{array}{l}\text { FDMS } \\
\left(\mathrm{m}^{3} / \mathrm{kg}\right)\end{array}$ & $\begin{array}{c}\text { ARM } \\
\left(\mathrm{Am}^{2} / \mathrm{kg}\right)\end{array}$ & $\begin{array}{c}\text { IRM1.2 } \\
\left(\mathrm{Am}^{2} / \mathrm{kg}\right) \\
\end{array}$ & $\begin{array}{l}\text { IRM -0.3 } \\
\left(\mathrm{Am}^{2} / \mathrm{kg}\right) \\
\end{array}$ & $\begin{array}{c}\text { HIRM } \\
\left(\mathrm{Am}^{2} / \mathrm{kg}\right)\end{array}$ & S \\
\hline $493 / 0-1$ & $4.87 \mathrm{E}-07$ & $4.81 \mathrm{E}-07$ & 1.31 & 6.39E-09 & $1.37 \mathrm{E}-04$ & $6.91 \mathrm{E}-03$ & $5.83 E-03$ & $5.44 \mathrm{E}-04$ & 0.84 \\
\hline $493 / 1-2$ & 4.98E-07 & 4.91E-07 & 1.51 & 7.54E-09 & 1.59E-04 & 7.23E-03 & $6.12 \mathrm{E}-03$ & 5.55E-04 & 0.85 \\
\hline $493 / 2-5$ & 4.30E-07 & $4.42 \mathrm{E}-07$ & -2.85 & $-1.22 \mathrm{E}-08$ & $1.61 \mathrm{E}-04$ & $6.44 \mathrm{E}-03$ & $5.49 \mathrm{E}-03$ & $4.79 \mathrm{E}-04$ & 0.85 \\
\hline 493/Bedrock & n.d. & n.d. & n.d. & n.d. & n.d. & n.d. & n.d. & n.d. & n.d. \\
\hline 29/0-1 & $2.54 \mathrm{E}-07$ & $2.50 \mathrm{E}-07$ & 1.87 & 4.75E-09 & 7.14E-05 & $3.51 E-03$ & $3.05 E-03$ & $2.27 \mathrm{E}-04$ & 0.87 \\
\hline 29/1-2 & 2.31E-07 & 2.27E-07 & 1.79 & 4.14E-09 & 7.55E-05 & 3.45E-03 & 3.03E-03 & 2.11E-04 & 0.88 \\
\hline $29 / 2-5$ & $1.36 \mathrm{E}-07$ & 1.34E-07 & 1.46 & 1.98E-09 & $5.45 \mathrm{E}-05$ & $2.10 \mathrm{E}-03$ & $2.07 \mathrm{E}-03$ & 1.15E-05 & 0.99 \\
\hline 29/Bedrock & 5.76E-09 & 9.68E-09 & -67.99 & $-3.92 \mathrm{E}-09$ & 5.31E-06 & 1.42E-04 & 7.63E-05 & $3.29 \mathrm{E}-05$ & 0.54 \\
\hline $55 / 0-1$ & 3.89E-07 & 3.82E-07 & 1.88 & 7.31E-09 & 8.51E-05 & $5.02 \mathrm{E}-03$ & 4.54E-03 & 2.38E-04 & 0.91 \\
\hline $55 / 1-2$ & 3.82E-07 & $3.76 \mathrm{E}-07$ & 1.45 & 5.52E-09 & 8.92E-05 & 4.70E-03 & 4.17E-03 & $2.68 \mathrm{E}-04$ & 0.89 \\
\hline $55 / 2-5$ & 2.82E-07 & 2.77E-07 & 1.79 & 5.04E-09 & 7.37E-05 & 3.43E-03 & 3.03E-03 & 2.02E-04 & 0.88 \\
\hline 55/Bedrock & 7.27E-09 & 1.09E-08 & -50.03 & $-3.64 \mathrm{E}-09$ & 2.18E-06 & 1.11E-04 & $1.55 E-05$ & 4.77E-05 & 0.14 \\
\hline $54 / 0-1$ & $2.43 \mathrm{E}-07$ & 2.36E-07 & 2.76 & 6.69E-09 & 6.34E-05 & 3.56E-03 & 3.07E-03 & $2.45 \mathrm{E}-04$ & 0.86 \\
\hline $54 / 1-2$ & 1.90E-07 & 1.84E-07 & 3.17 & 6.03E-09 & 7.00E-05 & 3.01E-03 & 2.02E-03 & 4.94E-04 & 0.67 \\
\hline $54 / 2-5$ & 8.62E-08 & 8.63E-08 & -0.18 & $-1.55 E-10$ & 3.98E-05 & 1.34E-03 & 7.70E-04 & 2.84E-04 & 0.58 \\
\hline 54/Bedrock & 2.36E-08 & 2.67E-08 & -13.13 & $-3.10 \mathrm{E}-09$ & 2.97E-06 & 3.76E-04 & 2.07E-05 & 1.78E-04 & 0.06 \\
\hline $53 / 0-1$ & 1.61E-07 & $1.60 \mathrm{E}-07$ & 1.01 & 1.63E-09 & 4.31E-05 & 2.26E-03 & 1.94E-03 & $1.58 \mathrm{E}-04$ & 0.86 \\
\hline $53 / 1-2$ & $1.25 \mathrm{E}-07$ & $1.22 \mathrm{E}-07$ & 3.11 & 3.90E-09 & 5.21E-05 & 1.82E-03 & $1.58 \mathrm{E}-03$ & 1.19E-04 & 0.87 \\
\hline $53 / 2-5$ & $1.92 \mathrm{E}-07$ & 1.86E-07 & 2.90 & 5.56E-09 & 5.83E-05 & 3.33E-03 & 2.83E-03 & $2.46 \mathrm{E}-04$ & 0.85 \\
\hline 53/Bedrock & 2.92E-09 & 5.78E-09 & -98.30 & $-2.87 \mathrm{E}-09$ & 4.29E-06 & 1.19E-04 & 1.00E-04 & 9.19E-06 & 0.85 \\
\hline $51 / 0-1$ & 2.15E-07 & 2.07E-07 & 3.95 & 8.50E-09 & 8.87E-05 & 2.35E-03 & 1.96E-03 & 1.98E-04 & 0.83 \\
\hline $51 / 1-2$ & 1.67E-07 & 1.61E-07 & 3.63 & 6.07E-09 & 8.60E-05 & 2.09E-03 & 1.75E-03 & $1.66 \mathrm{E}-04$ & 0.84 \\
\hline $51 / 2-5$ & 1.69E-07 & 1.61E-07 & 4.41 & 7.45E-09 & 8.37E-05 & 1.85E-03 & 1.70E-03 & 7.65E-05 & 0.92 \\
\hline 51/Bedrock & 5.46E-09 & 8.96E-09 & -64.29 & $-3.51 E-09$ & 2.19E-06 & 8.37E-05 & 7.68E-05 & $3.44 \mathrm{E}-06$ & 0.92 \\
\hline $50 / 0-1$ & 6.91E-08 & $6.78 \mathrm{E}-08$ & 1.95 & 1.35E-09 & 3.15E-05 & 1.06E-03 & 7.97E-04 & 1.31E-04 & 0.75 \\
\hline $50 / 1-2$ & $5.18 \mathrm{E}-08$ & $5.28 \mathrm{E}-08$ & -1.79 & $-9.27 \mathrm{E}-10$ & $2.79 \mathrm{E}-05$ & 8.52E-04 & 5.87E-04 & 1.32E-04 & 0.69 \\
\hline $50 / 2-5$ & 5.17E-08 & 5.19E-08 & -0.33 & $-1.68 \mathrm{E}-10$ & 2.13E-05 & 4.15E-04 & 3.00E-04 & $5.73 E-05$ & 0.72 \\
\hline 50/Bedrock & 2.94E-09 & 6.76E-09 & -129.50 & $-3.81 E-09$ & 2.40E-06 & 1.12E-04 & 5.85E-05 & $2.66 \mathrm{E}-05$ & 0.52 \\
\hline $49 / 0-1$ & 8.78E-08 & 8.66E-08 & 1.37 & 1.20E-09 & 3.13E-05 & 9.80E-04 & 8.83E-04 & 4.85E-05 & 0.90 \\
\hline $49 / 1-2$ & 6.83E-08 & 7.07E-08 & -3.53 & $-2.41 \mathrm{E}-09$ & 2.45E-05 & 6.97E-04 & 5.53E-04 & $7.20 \mathrm{E}-05$ & 0.79 \\
\hline $49 / 2-5$ & 6.03E-08 & $6.20 \mathrm{E}-08$ & -2.85 & $-1.72 \mathrm{E}-09$ & 1.05E-05 & 6.57E-04 & 5.39E-04 & 5.87E-05 & 0.82 \\
\hline 49/Bedrock & 2.26E-09 & 5.00E-09 & -121.09 & $-2.74 \mathrm{E}-09$ & 3.69E-06 & 1.85E-04 & 1.66E-04 & 9.30E-06 & 0.90 \\
\hline
\end{tabular}


Table 4. Magnetic properties.-Continued

Magnetic properties for $<2$-mm-size fraction and bulk bedrock-Continued.

\begin{tabular}{|c|c|c|c|c|c|c|c|c|c|}
\hline Sample no. & $\begin{array}{c}\text { MSIf } \\
\left(\mathrm{m}^{3} / \mathrm{kg}\right)\end{array}$ & $\begin{array}{c}\text { MShf } \\
\left(\mathrm{m}^{3} / \mathrm{kg}\right)\end{array}$ & $\begin{array}{c}\text { FDMS } \\
(\%)\end{array}$ & $\begin{array}{l}\text { FDMS } \\
\left(\mathrm{m}^{3} / \mathrm{kg}\right)\end{array}$ & $\begin{array}{c}\text { ARM } \\
\left(\mathrm{Am}^{2} / \mathrm{kg}\right)\end{array}$ & $\begin{array}{c}\text { IRM1.2 } \\
\left(\mathrm{Am}^{2} / \mathrm{kg}\right)\end{array}$ & $\begin{array}{l}\text { IRM -0.3 } \\
\left(\mathrm{Am}^{2} / \mathrm{kg}\right)\end{array}$ & $\begin{array}{c}\text { HIRM } \\
\left(\mathrm{Am}^{2} / \mathrm{kg}\right)\end{array}$ & $\mathrm{S}$ \\
\hline $48 / 0-1$ & $9.86 \mathrm{E}-08$ & $1.01 \mathrm{E}-07$ & -2.39 & $-2.36 \mathrm{E}-09$ & 1.47E-05 & $9.33 \mathrm{E}-04$ & $9.24 \mathrm{E}-04$ & $4.63 \mathrm{E}-06$ & 0.99 \\
\hline $48 / 1-2$ & 6.68E-08 & 6.60E-08 & 1.15 & 7.70E-10 & 1.12E-05 & 6.57E-04 & 5.76E-04 & 4.02E-05 & 0.88 \\
\hline $48 / 2-5$ & 7.22E-08 & 7.68E-08 & -6.39 & $-4.62 E-09$ & 2.53E-05 & 6.47E-04 & 5.66E-04 & 4.02E-05 & 0.88 \\
\hline 48/Bedrock & $5.00 \mathrm{E}-08$ & $5.27 \mathrm{E}-08$ & -5.21 & $-2.61 \mathrm{E}-09$ & $3.22 \mathrm{E}-06$ & 1.31E-04 & $2.25 \mathrm{E}-05$ & 5.44E-05 & 0.17 \\
\hline $47 / 0-1$ & 2.03E-07 & 1.99E-07 & 1.94 & 3.93E-09 & 6.90E-05 & 2.93E-03 & 2.37E-03 & 2.77E-04 & 0.81 \\
\hline $47 / 1-2$ & 1.77E-07 & $1.72 \mathrm{E}-07$ & 3.23 & 5.73E-09 & 5.52E-05 & 2.63E-03 & 2.21E-03 & 2.10E-04 & 0.84 \\
\hline $47 / 2-5$ & 1.17E-07 & 1.18E-07 & -1.11 & $-1.30 \mathrm{E}-09$ & 2.93E-05 & 1.60E-03 & 1.22E-03 & $1.89 \mathrm{E}-04$ & 0.76 \\
\hline 47/Bedrock & 4.06E-08 & 4.40E-08 & -8.44 & $-3.43 E-09$ & 7.04E-06 & 2.09E-04 & 1.66E-04 & 2.13E-05 & 0.80 \\
\hline $30 / 0-1$ & 1.70E-07 & 1.65E-07 & 2.72 & 4.61E-09 & 4.83E-05 & 3.49E-03 & 3.06E-03 & 2.17E-04 & 0.88 \\
\hline $30 / 1-2$ & 1.46E-07 & $1.45 \mathrm{E}-07$ & 0.29 & $4.28 \mathrm{E}-10$ & 4.46E-05 & 2.06E-03 & 1.84E-03 & 1.12E-04 & 0.89 \\
\hline $30 / 2-5$ & 4.44E-08 & 4.98E-08 & -12.26 & $-5.44 \mathrm{E}-09$ & 2.57E-05 & 6.41E-04 & 5.37E-04 & 5.20E-05 & 0.84 \\
\hline 30/Bedrock & $4.20 \mathrm{E}-09$ & 8.01E-09 & -90.88 & $-3.81 E-09$ & $2.94 \mathrm{E}-06$ & 6.83E-05 & 5.67E-05 & 5.79E-06 & 0.83 \\
\hline $46 / 0-1$ & 3.34E-08 & 3.59E-08 & -7.40 & $-2.47 \mathrm{E}-09$ & 2.24E-05 & 3.81E-04 & 3.43E-04 & 1.92E-05 & 0.90 \\
\hline $46 / 1-2$ & 2.71E-08 & 2.68E-08 & 1.06 & 2.88E-10 & 2.01E-05 & 3.38E-04 & 3.68E-04 & -1.50E-05 & 1.09 \\
\hline $46 / 2-5$ & $4.21 \mathrm{E}-08$ & 4.50E-08 & -6.81 & $-2.87 \mathrm{E}-09$ & 1.79E-05 & 5.66E-04 & 5.18E-04 & 2.45E-05 & 0.91 \\
\hline 46/Bedrock & n.d. & n.d. & n.d. & n.d. & n.d. & n.d. & n.d. & n.d. & n.d. \\
\hline $45 / 0-1$ & 7.25E-08 & 7.38E-08 & -1.78 & $-1.29 \mathrm{E}-09$ & 1.96E-05 & 8.39E-04 & 7.88E-04 & 2.59E-05 & 0.94 \\
\hline $45 / 1-2$ & 6.58E-08 & 6.84E-08 & -4.03 & $-2.65 E-09$ & 2.45E-05 & 6.10E-04 & 5.40E-04 & 3.51E-05 & 0.89 \\
\hline $45 / 2-5$ & 4.84E-08 & 4.67E-08 & 3.44 & 1.66E-09 & 2.14E-05 & 5.17E-04 & 4.70E-04 & 2.31E-05 & 0.91 \\
\hline 45/Bedrock & 3.47E-09 & 8.68E-09 & -149.70 & $-5.20 \mathrm{E}-09$ & 3.51E-06 & 8.95E-05 & 7.62E-05 & 6.65E-06 & 0.85 \\
\hline $44 / 0-1$ & 9.30E-08 & $9.60 \mathrm{E}-08$ & -3.29 & $-3.06 E-09$ & 3.17E-05 & 1.03E-03 & 8.08E-04 & 1.11E-04 & 0.78 \\
\hline $44 / 1-2$ & 6.63E-08 & 6.87E-08 & -3.59 & $-2.38 E-09$ & 1.61E-05 & 7.89E-04 & 6.64E-04 & $6.26 \mathrm{E}-05$ & 0.84 \\
\hline $44 / 2-5$ & 5.18E-08 & 5.52E-08 & -6.72 & $-3.48 E-09$ & 1.33E-05 & 6.45E-04 & 5.21E-04 & 6.19E-05 & 0.81 \\
\hline 44/Bedrock & 3.21E-09 & 7.26E-09 & -126.29 & $-4.05 E-09$ & 3.38E-06 & 5.96E-05 & 5.14E-05 & 4.07E-06 & 0.86 \\
\hline $05 U-1 / 0-1$ & 1.02E-07 & $1.02 \mathrm{E}-07$ & -0.26 & $-2.69 \mathrm{E}-10$ & 4.36E-05 & 1.77E-03 & 1.32E-03 & $2.26 \mathrm{E}-04$ & 0.75 \\
\hline $05 U-1 / 1-2$ & 3.60E-08 & 3.77E-08 & -4.76 & -1.71E-09 & 2.59E-05 & 6.36E-04 & 4.39E-04 & 9.85E-05 & 0.69 \\
\hline 05U-1/Bedrock & 4.34E-09 & 6.68E-09 & -53.80 & $-2.34 \mathrm{E}-09$ & 5.80E-06 & $1.59 \mathrm{E}-04$ & 6.27E-05 & 4.82E-05 & 0.39 \\
\hline $05 U-2 / 0-1$ & 1.13E-07 & $1.12 \mathrm{E}-07$ & 0.94 & 1.06E-09 & 6.21E-05 & 1.95E-03 & $1.42 \mathrm{E}-03$ & 2.68E-04 & 0.73 \\
\hline $05 U-2 / 1-2$ & 9.65E-08 & $9.62 \mathrm{E}-08$ & 0.26 & $2.55 \mathrm{E}-10$ & 5.59E-05 & 1.70E-03 & $1.22 \mathrm{E}-03$ & 2.41E-04 & 0.72 \\
\hline $05 U-2 / 2-5$ & 4.51E-08 & 4.74E-08 & -5.16 & $-2.33 E-09$ & 2.47E-05 & 8.40E-04 & 5.29E-04 & $1.55 \mathrm{E}-04$ & 0.63 \\
\hline 05U-2/Bedrock & 4.36E-09 & 7.35E-09 & -68.69 & $-2.99 E-09$ & 6.45E-06 & $1.54 \mathrm{E}-04$ & 6.94E-05 & 4.22E-05 & 0.45 \\
\hline $21 / 0-1$ & 8.91E-08 & 8.94E-08 & -0.36 & $-3.23 E-10$ & 3.01E-05 & 1.47E-03 & 1.01E-03 & 2.27E-04 & 0.69 \\
\hline $21 / 1-2$ & 6.21E-08 & 6.09E-08 & 1.88 & 1.17E-09 & 2.71E-05 & 1.05E-03 & 7.09E-04 & 1.72E-04 & 0.67 \\
\hline $21 / 2-5$ & 5.97E-08 & 6.34E-08 & -6.19 & $-3.70 \mathrm{E}-09$ & 2.46E-05 & 9.52E-04 & 6.26E-04 & 1.63E-04 & 0.66 \\
\hline 21/Bedrock & $1.44 \mathrm{E}-08$ & 1.69E-08 & -16.79 & $-2.42 \mathrm{E}-09$ & 8.04E-06 & 5.79E-04 & 2.04E-04 & 1.87E-04 & 0.35 \\
\hline
\end{tabular}


Table 4. Magnetic properties.-Continued

Magnetic properties for $<63-\mu \mathrm{m}$-size fraction.

\begin{tabular}{|c|c|c|c|c|c|c|c|c|c|}
\hline Sample no. & $\begin{array}{c}\text { MSlf } \\
\left(\mathrm{m}^{3} / \mathrm{kg}\right)\end{array}$ & $\begin{array}{c}\text { MShf } \\
\left(\mathrm{m}^{3} / \mathrm{kg}\right)\end{array}$ & $\begin{array}{c}\text { FDMS } \\
(\%)\end{array}$ & $\begin{array}{c}\text { FDMS } \\
\left(\mathrm{m}^{3} / \mathrm{kg}\right)\end{array}$ & $\begin{array}{c}\text { ARM } \\
\left(\mathrm{Am}^{2} / \mathrm{kg}\right)\end{array}$ & $\begin{array}{c}\text { IRM1.2 } \\
\left(\mathrm{Am}^{2} / \mathrm{kg}\right)\end{array}$ & $\begin{array}{l}\text { IRM -0.3 } \\
\left(\mathrm{Am}^{2} / \mathrm{kg}\right)\end{array}$ & $\begin{array}{c}\text { HIRM } \\
\left(\mathrm{Am}^{2} / \mathrm{kg}\right)\end{array}$ & $\mathrm{S}$ \\
\hline $493 / 0-1$ & 1.37E-06 & $1.36 \mathrm{E}-06$ & 1.37 & 1.88E-08 & $2.93 \mathrm{E}-04$ & $1.92 \mathrm{E}-02$ & 1.70E-02 & $1.11 \mathrm{E}-03$ & 0.88 \\
\hline $493 / 1-2$ & 1.35E-06 & 1.33E-06 & 1.79 & $2.42 \mathrm{E}-08$ & 3.35E-04 & 1.96E-02 & 1.71E-02 & $1.22 \mathrm{E}-03$ & 0.88 \\
\hline $493 / 2-5$ & $1.04 \mathrm{E}-06$ & 1.02E-06 & 1.86 & 1.93E-08 & 2.59E-04 & 1.49E-02 & 1.30E-02 & 9.34E-04 & 0.87 \\
\hline $29 / 0-1$ & 1.10E-06 & $1.08 \mathrm{E}-06$ & 1.78 & $1.95 \mathrm{E}-08$ & 2.81E-04 & 1.56E-02 & 1.41E-02 & 7.69E-04 & 0.90 \\
\hline $29 / 1-2$ & 1.03E-06 & $1.01 \mathrm{E}-06$ & 2.29 & $2.36 \mathrm{E}-08$ & 2.45E-04 & 1.46E-02 & 1.30E-02 & 8.19E-04 & 0.89 \\
\hline $29 / 2-5$ & 5.87E-07 & 5.73E-07 & 2.39 & $1.41 \mathrm{E}-08$ & 1.56E-04 & 8.85E-03 & 7.69E-03 & $5.84 \mathrm{E}-04$ & 0.87 \\
\hline $55 / 0-1$ & $1.40 \mathrm{E}-06$ & 1.37E-06 & 2.00 & $2.79 \mathrm{E}-08$ & 2.58E-04 & 1.82E-02 & 1.62E-02 & $9.62 \mathrm{E}-04$ & 0.89 \\
\hline $55 / 1-2$ & $1.25 \mathrm{E}-06$ & 1.23E-06 & 1.88 & $2.36 \mathrm{E}-08$ & 2.94E-04 & 1.67E-02 & 1.48E-02 & $9.49 \mathrm{E}-04$ & 0.89 \\
\hline $55 / 2-5$ & 1.18E-06 & 1.16E-06 & 2.34 & 2.76E-08 & 2.27E-04 & 1.55E-02 & 1.38E-02 & 8.35E-04 & 0.89 \\
\hline $54 / 0-1$ & $1.01 \mathrm{E}-06$ & 9.89E-07 & 2.38 & $2.41 \mathrm{E}-08$ & 2.36E-04 & 1.43E-02 & 1.23E-02 & 9.97E-04 & 0.86 \\
\hline $54 / 1-2$ & $6.65 \mathrm{E}-07$ & $6.45 \mathrm{E}-07$ & 3.12 & 2.07E-08 & 2.13E-04 & 1.01E-02 & 7.46E-03 & 1.33E-03 & 0.74 \\
\hline $54 / 2-5$ & 4.74E-07 & 4.59E-07 & 3.07 & 1.46E-08 & 1.51E-04 & 7.30E-03 & 5.05E-03 & 1.13E-03 & 0.69 \\
\hline $53 / 0-1$ & 9.01E-07 & 8.79E-07 & 2.39 & $2.15 \mathrm{E}-08$ & 1.85E-04 & 1.38E-02 & 1.19E-02 & 9.07E-04 & 0.87 \\
\hline $53 / 1-2$ & 8.51E-07 & 8.29E-07 & 2.57 & 2.18E-08 & 1.81E-04 & 1.31E-02 & 1.14E-02 & 8.37E-04 & 0.87 \\
\hline $53 / 2-5$ & $6.25 \mathrm{E}-07$ & 6.06E-07 & 2.93 & 1.83E-08 & 1.93E-04 & 1.05E-02 & 8.88E-03 & 7.90E-04 & 0.85 \\
\hline $51 / 0-1$ & 8.87E-07 & 8.49E-07 & 4.22 & $3.74 \mathrm{E}-08$ & 2.54E-04 & 1.24E-02 & 1.07E-02 & $8.21 E-04$ & 0.87 \\
\hline $51 / 1-2$ & 9.08E-07 & 8.76E-07 & 3.49 & 3.17E-08 & 2.66E-04 & 1.22E-02 & 1.06E-02 & 8.03E-04 & 0.87 \\
\hline $51 / 2-5$ & 7.78E-07 & 7.41E-07 & 4.74 & 3.69E-08 & 2.22E-04 & 1.08E-02 & $9.26 \mathrm{E}-03$ & 7.51E-04 & 0.86 \\
\hline $50 / 0-1$ & 6.16E-07 & 5.93E-07 & 3.79 & $2.34 \mathrm{E}-08$ & 1.43E-04 & 9.65E-03 & 7.88E-03 & 8.80E-04 & 0.82 \\
\hline $50 / 1-2$ & $6.15 \mathrm{E}-07$ & 5.93E-07 & 3.52 & 2.16E-08 & 1.45E-04 & $9.80 \mathrm{E}-03$ & 7.81E-03 & $9.99 \mathrm{E}-04$ & 0.80 \\
\hline $50 / 2-5$ & 6.68E-07 & $6.45 \mathrm{E}-07$ & 3.45 & 2.31E-08 & 1.52E-04 & 1.07E-02 & 8.65E-03 & $1.04 \mathrm{E}-03$ & 0.81 \\
\hline $49 / 0-1$ & 6.82E-07 & 6.64E-07 & 2.73 & $1.86 \mathrm{E}-08$ & 1.03E-04 & 7.63E-03 & 6.51E-03 & 5.58E-04 & 0.85 \\
\hline $49 / 1-2$ & 6.36E-07 & $6.27 \mathrm{E}-07$ & 1.43 & 9.09E-09 & 9.76E-05 & 6.90E-03 & 5.90E-03 & 5.00E-04 & 0.86 \\
\hline $49 / 2-5$ & n.d. & n.d. & n.d. & n.d. & n.d. & n.d. & n.d. & n.d. & n.d. \\
\hline $48 / 0-1$ & $4.72 \mathrm{E}-07$ & 4.64E-07 & 1.85 & 8.74E-09 & 7.15E-05 & $4.62 \mathrm{E}-03$ & 4.09E-03 & 2.64E-04 & 0.89 \\
\hline $48 / 1-2$ & 4.18E-07 & 4.17E-07 & 0.23 & $9.61 \mathrm{E}-10$ & 5.92E-05 & 3.83E-03 & 3.39E-03 & 2.17E-04 & 0.89 \\
\hline $48 / 2-5$ & $3.42 E-07$ & 3.32E-07 & 2.98 & $1.02 \mathrm{E}-08$ & 4.62E-05 & 3.15E-03 & 2.78E-03 & 1.83E-04 & 0.88 \\
\hline $47 / 0-1$ & 6.02E-07 & 5.85E-07 & 2.71 & 1.63E-08 & 1.82E-04 & 8.44E-03 & 7.14E-03 & 6.51E-04 & 0.85 \\
\hline $47 / 1-2$ & 5.48E-07 & 5.37E-07 & 2.12 & 1.16E-08 & 1.43E-04 & 7.66E-03 & 6.44E-03 & 6.09E-04 & 0.84 \\
\hline $47 / 2-5$ & 3.61E-07 & $3.56 \mathrm{E}-07$ & 1.35 & 4.89E-09 & 8.39E-05 & 4.88E-03 & 3.82E-03 & 5.31E-04 & 0.78 \\
\hline $30 / 0-1$ & 6.02E-07 & 5.93E-07 & 1.55 & 9.33E-09 & 1.33E-04 & 8.40E-03 & 7.71E-03 & $3.45 E-04$ & 0.92 \\
\hline $30 / 1-2$ & 4.63E-07 & 4.48E-07 & 3.30 & 1.53E-08 & 1.16E-04 & $6.78 \mathrm{E}-03$ & $6.06 \mathrm{E}-03$ & $3.58 \mathrm{E}-04$ & 0.89 \\
\hline $30 / 2-5$ & 1.59E-07 & $1.59 \mathrm{E}-07$ & 0.08 & $1.20 \mathrm{E}-10$ & 5.13E-05 & $2.17 \mathrm{E}-03$ & 1.85E-03 & 1.62E-04 & 0.85 \\
\hline $46 / 0-1$ & 5.64E-07 & 5.52E-07 & 2.00 & 1.13E-08 & 1.37E-04 & 7.47E-03 & 6.78E-03 & $3.45 E-04$ & 0.91 \\
\hline $46 / 1-2$ & $6.25 \mathrm{E}-07$ & $6.15 \mathrm{E}-07$ & 1.65 & 1.03E-08 & 1.29E-04 & 8.01E-03 & 7.38E-03 & 3.15E-04 & 0.92 \\
\hline $46 / 2-5$ & $4.75 \mathrm{E}-07$ & $4.68 \mathrm{E}-07$ & 1.52 & 7.22E-09 & 1.09E-04 & $6.22 \mathrm{E}-03$ & $5.68 \mathrm{E}-03$ & $2.66 \mathrm{E}-04$ & 0.91 \\
\hline $45 / 0-1$ & 7.66E-07 & 7.52E-07 & 1.78 & 1.36E-08 & 1.27E-04 & 9.97E-03 & 9.13E-03 & 4.20E-04 & 0.92 \\
\hline $45 / 1-2$ & 7.26E-07 & 7.10E-07 & 2.15 & 1.56E-08 & 1.16E-04 & $9.23 \mathrm{E}-03$ & $8.37 \mathrm{E}-03$ & $4.28 \mathrm{E}-04$ & 0.91 \\
\hline $45 / 2-5$ & $6.62 \mathrm{E}-07$ & $6.48 \mathrm{E}-07$ & 2.06 & 1.37E-08 & 1.13E-04 & 8.53E-03 & 7.77E-03 & 3.81E-04 & 0.91 \\
\hline $44 / 0-1$ & 3.92E-07 & 3.82E-07 & 2.42 & $9.49 \mathrm{E}-09$ & 7.83E-05 & $5.14 \mathrm{E}-03$ & $4.21 \mathrm{E}-03$ & 4.64E-04 & 0.82 \\
\hline $44 / 1-2$ & 3.46E-07 & 3.38E-07 & 2.14 & 7.40E-09 & 7.18E-05 & $4.48 \mathrm{E}-03$ & $3.65 E-03$ & 4.11E-04 & 0.82 \\
\hline $44 / 2-5$ & 3.15E-07 & 3.08E-07 & 2.13 & 6.69E-09 & 6.50E-05 & 3.99E-03 & 3.31E-03 & 3.38E-04 & 0.83 \\
\hline $05 U-1 / 0-1$ & 4.53E-07 & 4.46E-07 & 1.68 & 7.62E-09 & 1.26E-04 & 7.37E-03 & 5.63E-03 & 8.67E-04 & 0.76 \\
\hline $05 U-1 / 1-2$ & 2.84E-07 & 2.81E-07 & 1.26 & 3.58E-09 & 9.34E-05 & 4.78E-03 & $3.47 \mathrm{E}-03$ & $6.58 \mathrm{E}-04$ & 0.72 \\
\hline $05 U-2 / 0-1$ & 3.77E-07 & 3.70E-07 & 1.82 & 6.85E-09 & 1.37E-04 & 6.08E-03 & 4.59E-03 & 7.47E-04 & 0.75 \\
\hline $05 U-2 / 1-2$ & $3.52 \mathrm{E}-07$ & $3.43 \mathrm{E}-07$ & 2.73 & $9.60 \mathrm{E}-09$ & 1.26E-04 & $5.72 \mathrm{E}-03$ & $4.25 \mathrm{E}-03$ & 7.33E-04 & 0.74 \\
\hline $05 U-2 / 2-5$ & 2.24E-07 & 2.23E-07 & 0.67 & 1.50E-09 & 6.85E-05 & 3.72E-03 & 2.47E-03 & $6.23 \mathrm{E}-04$ & 0.67 \\
\hline $21 / 0-1$ & 3.81E-07 & $3.78 \mathrm{E}-07$ & 0.97 & 3.69E-09 & 7.53E-05 & $5.50 \mathrm{E}-03$ & $3.84 \mathrm{E}-03$ & $8.29 \mathrm{E}-04$ & 0.70 \\
\hline $21 / 1-2$ & 3.32E-07 & $3.28 \mathrm{E}-07$ & 1.32 & 4.40E-09 & 7.02E-05 & 4.97E-03 & 3.32E-03 & $8.25 E-04$ & 0.67 \\
\hline $21 / 2-5$ & 3.11E-07 & 3.06E-07 & 1.32 & 4.11E-09 & 6.48E-05 & 4.66E-03 & 3.09E-03 & 7.85E-04 & 0.66 \\
\hline
\end{tabular}


Table 5. Geochemical data.

This table contains geochemical data obtained by ICP-AES (inductively coupled plasma-atomic emission spectrometry), ICP-MS (inductively coupled plasma-mass spectrometry), and XRF (X-ray fluorescence) techniques (Litche and others, 1987). All analyses were performed at the USGS Geologic Division geochemistry laboratory in Denver, Colorado, and are reported in parts per million (ppm) or percent (\%).

[Sample no., Unique sample number (for example, 44/0-1); presented as site designation (44), and depth in centimeters $(0-1)$.

n.d., not determined; ${ }^{*}, \mathrm{Zr}$ (ppm) and Ti (\%) obtained by XRF]

ICP-AES and XRF data

\begin{tabular}{|c|c|c|c|c|c|c|c|c|c|c|c|}
\hline Sample no. & Al \% & $\mathrm{Ca} \%$ & $\mathrm{Fe} \%$ & K \% & Mg \% & $\mathrm{Na} \%$ & P \% & Mn ppm & Nd ppm & *Zr ppm & ${ }^{*} \mathrm{Ti} \%$ \\
\hline $493 / 0-1$ & 5.98 & 1.46 & 2.91 & 2.00 & 0.975 & 1.21 & 0.0952 & 550 & 50.3 & 581 & 0.84 \\
\hline $493 / 1-2$ & 6.44 & 1.41 & 3.16 & 2.11 & 1.05 & 1.17 & 0.0982 & 572 & 43.6 & 562 & 0.84 \\
\hline $493 / 2-5$ & 6.64 & 1.35 & 3.26 & 2.16 & 1.04 & 1.07 & 0.0930 & 549 & 36.0 & 457 & 0.75 \\
\hline 493/Bedrock & 6.12 & 4.82 & 2.04 & 2.30 & 1.83 & 1.46 & 0.0686 & 304 & 22.5 & 77 & 0.24 \\
\hline 29/0-1 & 7.14 & 1.05 & 2.56 & 3.14 & 0.862 & 0.919 & 0.0661 & 394 & 29.3 & 485 & 0.72 \\
\hline $29 / 1-2$ & 7.39 & 0.933 & 2.46 & 3.11 & 0.778 & 0.867 & 0.0642 & 357 & 29.8 & 419 & 0.74 \\
\hline $29 / 2-5$ & 7.34 & 0.542 & 2.13 & 3.19 & 0.635 & 0.580 & 0.0366 & 273 & 26.5 & 284 & 0.63 \\
\hline 29/Bedrock & 1.73 & 0.0356 & 0.509 & 0.879 & 0.0861 & 0.0281 & 0.00782 & 61.3 & $<4$ & 59 & 0.09 \\
\hline $55 / 0-1$ & 5.11 & 1.83 & 2.68 & 2.24 & 1.35 & 0.878 & 0.0848 & 515 & 34.8 & 764 & 0.71 \\
\hline $55 / 1-2$ & 5.71 & 1.24 & 2.94 & 2.48 & 1.17 & 0.768 & 0.0893 & 411 & 32.0 & 638 & 0.80 \\
\hline $55 / 2-5$ & 5.47 & 1.04 & 2.76 & 2.38 & 0.985 & 0.743 & 0.0782 & 348 & 29.1 & 631 & 0.75 \\
\hline 55/Bedrock & 1.40 & 0.0672 & 1.08 & 1.47 & 0.0686 & 0.0352 & 0.0327 & 24.6 & $<4$ & 38 & 0.06 \\
\hline $54 / 0-1$ & 5.24 & 1.06 & 2.67 & 2.39 & 1.02 & 0.776 & 0.0839 & 461 & 30.3 & 399 & 0.63 \\
\hline $54 / 1-2$ & 6.06 & 0.697 & 3.15 & 2.71 & 0.977 & 0.524 & 0.104 & 345 & 29.4 & 233 & 0.66 \\
\hline $54 / 2-5$ & 5.60 & 0.573 & 2.79 & 2.62 & 0.837 & 0.426 & 0.0914 & 290 & 25.5 & 189 & 0.55 \\
\hline 54/Bedrock & 2.17 & 0.280 & 0.779 & 1.48 & 0.0690 & 0.0353 & 0.0571 & 135 & 5.46 & 52 & 0.06 \\
\hline $53 / 0-1$ & 5.61 & 0.692 & 2.01 & 3.08 & 0.498 & 0.843 & 0.0577 & 338 & 21.5 & 917 & 0.64 \\
\hline $53 / 1-2$ & 5.58 & 0.602 & 1.97 & 3.07 & 0.478 & 0.781 & 0.0515 & 329 & 20.8 & 869 & 0.68 \\
\hline $53 / 2-5$ & 7.95 & 0.435 & 2.09 & 2.83 & 0.467 & 0.620 & 0.0371 & 293 & 23.0 & 475 & 0.88 \\
\hline 53/Bedrock & 1.35 & 0.0716 & 0.473 & 0.620 & 0.0547 & 0.0248 & 0.0135 & 133 & $<4$ & 52 & 0.10 \\
\hline $51 / 0-1$ & 5.40 & 0.854 & 2.66 & 2.49 & 0.674 & 0.642 & 0.0648 & 693 & 28.5 & 1,273 & 0.80 \\
\hline $51 / 1-2$ & 5.52 & 0.931 & 2.77 & 2.51 & 0.697 & 0.630 & 0.0654 & 748 & 27.4 & 1,260 & 0.78 \\
\hline $51 / 2-5$ & 5.40 & 1.27 & 2.64 & 2.43 & 0.694 & 0.552 & 0.0544 & 714 & 26.3 & 1,107 & 0.75 \\
\hline 51/Bedrock & 0.350 & 41.5 & 0.183 & 0.251 & 0.451 & 0.0302 & 0.0105 & 348 & $<4$ & 10 & 0.02 \\
\hline $50 / 0-1$ & 4.20 & 1.13 & 2.05 & 2.53 & 0.569 & 0.528 & 0.0794 & 826 & 22.8 & 1,214 & 0.72 \\
\hline $50 / 1-2$ & 4.00 & 1.03 & 2.00 & 2.47 & 0.526 & 0.496 & 0.0733 & 846 & 22.9 & 1,531 & 0.80 \\
\hline $50 / 2-5$ & 4.14 & 1.10 & 2.12 & 2.51 & 0.551 & 0.520 & 0.0760 & 875 & 24.4 & 1,409 & 0.77 \\
\hline 50/Bedrock & 0.597 & 7.74 & 0.412 & 0.599 & 0.0467 & 0.0172 & 0.00826 & 392 & $<4$ & 27 & 0.03 \\
\hline $49 / 0-1$ & 4.28 & 0.975 & 1.56 & 2.42 & 0.688 & 0.464 & 0.0722 & 355 & 28.4 & 1,772 & 0.71 \\
\hline $49 / 1-2$ & 4.48 & 0.938 & 1.50 & 2.44 & 0.671 & 0.442 & 0.0684 & 334 & 24.5 & 1,543 & 0.60 \\
\hline $49 / 2-5$ & n.d. & n.d. & n.d. & n.d. & n.d. & n.d. & n.d. & n.d. & n.d. & n.d. & n.d. \\
\hline 49/Bedrock & 0.683 & 0.0744 & 0.315 & 0.421 & 0.0287 & 0.0172 & 0.00994 & 30.5 & $<4$ & 52 & 0.06 \\
\hline $48 / 0-1$ & 4.91 & 4.93 & 1.58 & 1.51 & 0.626 & 0.555 & 0.0768 & 426 & 24.0 & 1,229 & 0.67 \\
\hline $48 / 1-2$ & 4.51 & 5.31 & 1.41 & 1.51 & 0.546 & 0.561 & 0.0715 & 402 & 23.2 & 1,210 & 0.63 \\
\hline $48 / 2-5$ & 4.61 & 6.95 & 1.39 & 1.46 & 0.510 & 0.534 & 0.0674 & 386 & 25.6 & 1,147 & 0.65 \\
\hline 48/Bedrock & 1.72 & 1.02 & 7.37 & 0.109 & 0.0554 & 0.0504 & 0.0179 & 46.4 & 11.7 & 226 & 0.27 \\
\hline $47 / 0-1$ & 7.24 & 0.549 & 2.03 & 2.38 & 0.560 & 0.695 & 0.0441 & 288 & 23.6 & 592 & 0.73 \\
\hline $47 / 1-2$ & 7.49 & 0.519 & 2.03 & 2.38 & 0.555 & 0.668 & 0.0431 & 279 & 22.6 & 578 & 0.72 \\
\hline $47 / 2-5$ & 8.11 & 0.426 & 1.80 & 2.33 & 0.472 & 0.633 & 0.0256 & 221 & 21.9 & 410 & 0.73 \\
\hline 47/Bedrock & 1.53 & 0.0418 & 4.60 & 0.802 & 0.0855 & 0.0376 & 0.0491 & 245 & 6.33 & 49 & 0.07 \\
\hline
\end{tabular}


Table 5. Geochemical data.-Continued

ICP-AES and XRF data—continued.

\begin{tabular}{|c|c|c|c|c|c|c|c|c|c|c|c|}
\hline Sample no. & Al \% & $\mathrm{Ca} \%$ & Fe \% & K \% & Mg \% & $\mathrm{Na} \%$ & P \% & Mn ppm & Nd ppm & ${ }^{*} \mathrm{Zr}$ ppm & ${ }^{*} \mathrm{Ti} \%$ \\
\hline $30 / 0-1$ & 8.01 & 0.293 & 1.95 & 2.05 & 0.368 & 0.330 & 0.0576 & 169 & 21.8 & 662 & 0.74 \\
\hline $30 / 1-2$ & 9.62 & 0.271 & 2.04 & 2.00 & 0.376 & 0.301 & 0.0624 & 146 & 25.0 & 486 & 0.75 \\
\hline $30 / 2-5$ & 10.9 & 0.182 & 1.90 & 1.91 & 0.336 & 0.167 & 0.0676 & 99.4 & 22.0 & 256 & 0.58 \\
\hline 30/Bedrock & 1.58 & 0.0351 & 0.583 & 0.869 & 0.0542 & 0.0319 & 0.0138 & 33.8 & 6.44 & 95 & 0.12 \\
\hline $46 / 0-1$ & n.d. & n.d. & n.d. & n.d. & n.d. & n.d. & n.d. & n.d. & n.d. & n.d. & n.d. \\
\hline $46 / 1-2$ & n.d. & n.d. & n.d. & n.d. & n.d. & n.d. & n.d. & n.d. & n.d. & n.d. & n.d. \\
\hline $46 / 2-5$ & n.d. & n.d. & n.d. & n.d. & n.d. & n.d. & n.d. & n.d. & n.d. & n.d. & n.d. \\
\hline 46/Bedrock & n.d. & n.d. & n.d. & n.d. & n.d. & n.d. & n.d. & n.d. & n.d. & n.d. & n.d. \\
\hline $45 / 0-1$ & 4.60 & 0.420 & 1.42 & 3.17 & 0.320 & 0.337 & 0.0457 & 246 & 18.9 & 2,569 & 0.71 \\
\hline $45 / 1-2$ & 4.38 & 0.384 & 1.42 & 3.11 & 0.294 & 0.313 & 0.0443 & 245 & 18.6 & 2,159 & 0.59 \\
\hline $45 / 2-5$ & 5.12 & 0.403 & 1.34 & 3.26 & 0.307 & 0.305 & 0.0415 & 226 & 19.4 & 2,387 & 0.71 \\
\hline 45/Bedrock & 1.86 & 0.0424 & 0.335 & 1.20 & 0.0900 & 0.0433 & 0.00759 & 26.8 & $<4$ & 63 & 0.10 \\
\hline $44 / 0-1$ & 4.28 & 1.75 & 1.59 & 2.18 & 1.03 & 0.596 & 0.0754 & 325 & 30.4 & 1,514 & 0.61 \\
\hline $44 / 1-2$ & 4.23 & 1.74 & 1.49 & 2.20 & 0.994 & 0.598 & 0.0715 & 306 & 28.0 & 1,513 & 0.67 \\
\hline $44 / 2-5$ & 4.19 & 1.70 & 1.46 & 2.21 & 0.945 & 0.587 & 0.0692 & 296 & 29.1 & 1,554 & 0.71 \\
\hline 44/Bedrock & 2.02 & 0.350 & 0.232 & 1.14 & 0.0893 & 0.0344 & 0.00956 & 11.1 & 4.81 & 289 & 0.22 \\
\hline $05 U-1 / 0-1$ & 5.77 & 0.597 & 2.06 & 3.06 & 0.718 & 0.483 & 0.078 & 346 & 28.1 & 991 & 0.98 \\
\hline $05 U-1 / 1-2$ & 5.34 & 0.448 & 1.65 & 3.26 & 0.56 & 0.369 & 0.062 & 270 & 23.4 & 779 & 0.83 \\
\hline 05U-1/Bedrock & 1.51 & 0.375 & 0.302 & 1.5 & 0.0746 & 0.0456 & 0.00698 & 130 & $<4$ & 142 & 0.12 \\
\hline $05 U-2 / 0-1$ & 5.55 & 0.536 & 2.03 & 2.84 & 0.67 & 0.541 & 0.07 & 363 & 26.9 & 715 & 0.77 \\
\hline $05 U-2 / 1-2$ & 5.7 & 0.503 & 1.99 & 2.95 & 0.64 & 0.552 & 0.0664 & 357 & 24.8 & 628 & 0.75 \\
\hline $05 U-2 / 2-5$ & 5.31 & 0.366 & 1.72 & 2.97 & 0.555 & 0.487 & 0.0319 & 287 & 21.7 & 634 & 0.68 \\
\hline 05U-2/Bedrock & 1.65 & 0.0335 & 0.212 & 1.75 & 0.0604 & 0.0555 & 0.00557 & 35.2 & $<4$ & 62 & 0.09 \\
\hline $21 / 0-1$ & 4 & 3.2 & 1.7 & 1.9 & 0.83 & 0.67 & 0.055 & 380 & 23 & 903 & 0.65 \\
\hline $21 / 1-2$ & 3.3 & 2.4 & 1.4 & 1.8 & 0.64 & 0.55 & 0.04 & 300 & 17 & 822 & 0.60 \\
\hline $21 / 2-5$ & 3.8 & 2.5 & 1.5 & 2 & 0.69 & 0.67 & 0.047 & 340 & 19 & 899 & 0.63 \\
\hline 21/Bedrock & 1.7 & 0.64 & 0.32 & 1.3 & 0.17 & 0.086 & 0.013 & 62 & $<8$ & 174 & 0.12 \\
\hline
\end{tabular}


Table 5. Geochemical data.-Continued

ICP-MS data

\begin{tabular}{|c|c|c|c|c|c|c|c|c|c|c|c|c|}
\hline Sample no. & $\begin{array}{c}\mathrm{Ag} \\
(\mathrm{ppm})\end{array}$ & $\begin{array}{c}\mathrm{Al} \\
(\mathrm{ppm})\end{array}$ & $\begin{array}{c}\text { As } \\
\text { (ppm) }\end{array}$ & $\begin{array}{c}\mathrm{Ba} \\
(\mathrm{ppm})\end{array}$ & $\begin{array}{c}\mathrm{Be} \\
(\mathrm{ppm})\end{array}$ & $\begin{array}{c}\mathrm{Bi} \\
(\mathbf{p p m})\end{array}$ & $\begin{array}{l}\mathrm{Ca} \\
(\mathrm{ppm})\end{array}$ & $\begin{array}{c}\mathrm{Cd} \\
(\mathrm{ppm})\end{array}$ & $\begin{array}{c}\mathrm{Ce} \\
(\mathrm{ppm})\end{array}$ & $\begin{array}{c}\text { Co } \\
\text { (ppm) }\end{array}$ & $\begin{array}{c}\mathrm{Cr} \\
(\mathrm{ppm})\end{array}$ & $\begin{array}{c}\text { Cs } \\
\text { (ppm) }\end{array}$ \\
\hline $493 / 0-1$ & $<2$ & 69,600 & 8.9 & 903 & 2 & 0.3 & 17,100 & 0.3 & 204 & 9.8 & 56.8 & 6.8 \\
\hline 493/1-2 & $<2$ & 77,000 & 11 & 882 & 2.2 & 0.31 & 16,900 & 0.32 & 163 & 10.7 & 62.8 & 8.3 \\
\hline $493 / 2-5$ & $<2$ & 66,500 & 10.1 & 691 & 2 & 0.21 & 13,000 & 0.24 & 88 & 9.2 & 54.4 & 8.4 \\
\hline 493/Bedrock & $<2$ & 35,600 & 8.8 & 413 & 0.98 & $<0.06$ & 21,500 & 0.06 & 29 & 4 & 37 & 3.3 \\
\hline 29/0-1 & $<2$ & 89,600 & 11.4 & 954 & 1.8 & 0.27 & 11,300 & 0.28 & 79.2 & 7.8 & 54 & 5.3 \\
\hline $29 / 1-2$ & $<2$ & 95,800 & 12.1 & 985 & 1.9 & 0.26 & 10,200 & 0.24 & 80.2 & 7.6 & 49 & 5.5 \\
\hline $29 / 2-5$ & $<2$ & 98,300 & 13.6 & 956 & 1.8 & 0.15 & 5,760 & 0.11 & 65.3 & 6.5 & 42 & 5.8 \\
\hline 29/Bedrock & $<2$ & 18,900 & 2.4 & 225 & 0.46 & $<0.06$ & 314 & 0.01 & 10.1 & 0.94 & 12.8 & 1.1 \\
\hline 55/0-1 & $<2$ & 56,600 & 13.8 & 652 & 1.5 & 0.31 & 17,700 & 0.28 & 96.4 & 7 & 55.6 & 4.6 \\
\hline $55 / 1-2$ & $<2$ & 67,800 & 18.3 & 640 & 2 & 0.26 & 12,100 & 0.26 & 73.7 & 7.5 & 60.6 & 6.1 \\
\hline $55 / 2-5$ & $<2$ & 65,200 & 17.1 & 617 & 1.9 & 0.17 & 9,870 & 0.18 & 68.4 & 6.8 & 56.8 & 6.1 \\
\hline 55/Bedrock & $<2$ & 15,200 & 3.4 & 337 & 0.22 & $<0.06$ & 530 & 0.008 & 6.5 & 0.33 & 18.8 & 1 \\
\hline $54 / 0-1$ & $<2$ & 58,000 & 19.8 & 592 & 1.6 & 0.33 & 10,500 & 0.3 & 78.5 & 7 & 44.2 & 4.9 \\
\hline $54 / 1-2$ & $<2$ & 75,200 & 43 & 648 & 2.3 & 0.35 & 7,280 & 0.3 & 66.4 & 7.6 & 55.2 & 7.2 \\
\hline $54 / 2-5$ & $<2$ & 66,100 & 39 & 560 & 2 & 0.26 & 5,770 & 0.23 & 58.6 & 6.7 & 47.8 & 6.5 \\
\hline 54/Bedrock & $<2$ & 24,600 & 29.5 & 393 & 0.7 & $<0.06$ & 3,120 & 0.08 & 11.6 & 1.6 & 41.7 & 0.93 \\
\hline 53/0-1 & $<2$ & 65,500 & 6.4 & 942 & 1.3 & 0.31 & 6,340 & 0.23 & 48.8 & 5.8 & 47 & 4.9 \\
\hline $53 / 1-2$ & $<2$ & 70,900 & 6.9 & 1000 & 1.3 & 0.3 & 6,260 & 0.26 & 54.2 & 5.9 & 44.6 & 5.1 \\
\hline $53 / 2-5$ & $<2$ & 95,600 & 10.2 & 887 & 1.5 & 0.21 & 4,880 & 0.15 & 66.2 & 6.6 & 49.4 & 5.7 \\
\hline 53/Bedrock & $<2$ & 14,700 & 2.7 & 174 & 0.27 & $<0.06$ & 764 & 0.04 & 12 & 2.7 & 37.6 & 1.1 \\
\hline 51/0-1 & $<2$ & 56,800 & 9.8 & 583 & 1.6 & 0.25 & 8,020 & 0.29 & 61.6 & 8.6 & 53 & 5.5 \\
\hline $51 / 1-2$ & $<2$ & 56,900 & 9.2 & 593 & 1.6 & 0.25 & 8,070 & 0.29 & 61.4 & 8.6 & 54 & 5.5 \\
\hline $51 / 2-5$ & $<2$ & 58,600 & 10.3 & 582 & 1.8 & 0.24 & 12,200 & 0.31 & 65.2 & 9.2 & 51.4 & 5.9 \\
\hline 51/Bedrock & $<2$ & 3,200 & 1 & 19.5 & 0.18 & $<0.06$ & 454,000 & 0.16 & 3 & 0.18 & 4 & 0.33 \\
\hline $50 / 0-1$ & $<2$ & 38,900 & 5.6 & 492 & 1.1 & 0.27 & 9,620 & 0.28 & 52.3 & 4.6 & 58.3 & 2.9 \\
\hline $50 / 1-2$ & $<2$ & 36,600 & 5 & 477 & 1 & 0.26 & 8,280 & 0.26 & 59.6 & 4.4 & 48 & 2.6 \\
\hline $50 / 2-5$ & $<2$ & 36,800 & 5.2 & 473 & 1 & 0.31 & 8,680 & 0.34 & 51.4 & 4.5 & 54.1 & 2.7 \\
\hline 50/Bedrock & $<2$ & 5,920 & 1.6 & 106 & 0.17 & $<0.06$ & 81,700 & 0.05 & 4.4 & 0.68 & 40.4 & 0.38 \\
\hline $49 / 0-1$ & $<2$ & 46,000 & 6.1 & 692 & 1 & 0.26 & 9,520 & 0.24 & 59.4 & 4.3 & 37.4 & 3.1 \\
\hline $49 / 1-2$ & $<2$ & 42,700 & 4.9 & 664 & 0.87 & 0.22 & 7,460 & 0.21 & 48.3 & 3.6 & 40.8 & 2.9 \\
\hline $49 / 2-5$ & n.d. & n.d. & n.d. & n.d. & n.d. & n.d. & n.d. & n.d. & n.d. & n.d. & n.d. & n.d. \\
\hline 49/Bedrock & $<2$ & 7,510 & $<1$ & 100 & 0.15 & $<0.06$ & 363 & 0.01 & 7.2 & 0.38 & 19.3 & 0.41 \\
\hline $48 / 0-1$ & $<2$ & 48,800 & 6.7 & 390 & 1.3 & 0.23 & 46,700 & 0.18 & 48.6 & 3.8 & 32.8 & 2.8 \\
\hline $48 / 1-2$ & $<2$ & 45,200 & 5.8 & 381 & 1.2 & 0.2 & 50,300 & 0.15 & 47.9 & 3.4 & 33.4 & 2.6 \\
\hline $48 / 2-5$ & $<2$ & 45,900 & 6.5 & 352 & 1.2 & 0.2 & 64,100 & 0.14 & 53.8 & 3.4 & 29.6 & 2.5 \\
\hline 48/Bedrock & $<2$ & 16,900 & 80.2 & 166 & 1.2 & 0.37 & 9,660 & 0.12 & 20.9 & 0.82 & 14.8 & 0.22 \\
\hline $47 / 0-1$ & $<2$ & 83,100 & 18.8 & 602 & 1.8 & 0.38 & 5,380 & 0.31 & 54.5 & 5.9 & 44.3 & 5.1 \\
\hline $47 / 1-2$ & $<2$ & 85,200 & 19.1 & 608 & 1.9 & 0.42 & 5,100 & 0.32 & 54.5 & 5.8 & 48.8 & 5.3 \\
\hline $47 / 2-5$ & $<2$ & 102,000 & 20.6 & 575 & 2 & 0.27 & 4,470 & 0.17 & 52.4 & 5.2 & 45.3 & 5 \\
\hline 47/Bedrock & $<2$ & 14,500 & 273 & 217 & 1.6 & $<0.06$ & 461 & 0.32 & 8.6 & 2 & 14.9 & 1.2 \\
\hline $30 / 0-1$ & $<2$ & 107,000 & 233 & 671 & 1.5 & 0.24 & 3,260 & 0.26 & 62.7 & 5 & 41.3 & 5.4 \\
\hline $30 / 1-2$ & $<2$ & 115,000 & 294 & 540 & 1.6 & 0.27 & 2,930 & 0.22 & 63.4 & 5.1 & 38 & 5.5 \\
\hline $30 / 2-5$ & $<2$ & 129,000 & 303 & 544 & 1.7 & 0.19 & 2,250 & 0.14 & 53.5 & 4.2 & 33.8 & 5.8 \\
\hline 30/Bedrock & $<2$ & 15,900 & 59.2 & 341 & 0.34 & 0.07 & 409 & 0.03 & 14.9 & 0.58 & 33 & 1.3 \\
\hline $46 / 0-1$ & n.d. & n.d. & n.d. & n.d. & n.d. & n.d. & n.d. & n.d. & n.d. & n.d. & n.d. & n.d. \\
\hline $46 / 1-2$ & n.d. & n.d. & n.d. & n.d. & n.d. & n.d. & n.d. & n.d. & n.d. & n.d. & n.d. & n.d. \\
\hline $46 / 2-5$ & n.d. & n.d. & n.d. & n.d. & n.d. & n.d. & n.d. & n.d. & n.d. & n.d. & n.d. & n.d. \\
\hline 46/Bedrock & n.d. & n.d. & n.d. & n.d. & n.d. & n.d. & n.d. & n.d. & n.d. & n.d. & n.d. & n.d. \\
\hline $45 / 0-1$ & $<2$ & 42,000 & 19.6 & 675 & 0.82 & 0.12 & 2,820 & 0.1 & 29.2 & 2.9 & 43.4 & 4.4 \\
\hline $45 / 1-2$ & $<2$ & 33,300 & 14.2 & 616 & 0.59 & 0.07 & 1,540 & 0.06 & 17.8 & 2 & 25.9 & 3.6 \\
\hline $45 / 2-5$ & $<2$ & 48,500 & 20.7 & 723 & 0.82 & 0.11 & 2,770 & 0.09 & 29.2 & 2.7 & 41.4 & 4.7 \\
\hline 45/Bedrock & $<2$ & 20,600 & 4.6 & 293 & 0.41 & $<0.06$ & 263 & 0.01 & 8.1 & 0.85 & 17.7 & 2 \\
\hline $44 / 0-1$ & $<2$ & 44,600 & 8.8 & 550 & 1.3 & 0.14 & 14,300 & 0.14 & 47.5 & 3.8 & 41.2 & 3.7 \\
\hline $44 / 1-2$ & $<2$ & 48,400 & 9.4 & 558 & 1.5 & 0.15 & 17,400 & 0.14 & 58.7 & 4.2 & 41.8 & 4 \\
\hline $44 / 2-5$ & $<2$ & 46,600 & 9.8 & 532 & 1.5 & 0.13 & 17,000 & 0.11 & 56.9 & 4.1 & 41.3 & 3.7 \\
\hline 44/Bedrock & $<2$ & 20,600 & 2.2 & 233 & 0.77 & $<0.06$ & 3,120 & $<0.007$ & 11.8 & 0.62 & 21.2 & 1.8 \\
\hline $05 U-1 / 0-1$ & $<2$ & 60,300 & 19.6 & 518 & 1.6 & 0.44 & 6,080 & 0.42 & 81.7 & 6.6 & 45 & 4.8 \\
\hline $05 U-1 / 1-2$ & $<2$ & 57,000 & 15.6 & 494 & 1.4 & 0.4 & 4,670 & 0.47 & 53.8 & 5.4 & 33.5 & 4.3 \\
\hline 05U-1/Bedrock & $<2$ & 14,700 & 1.6 & 296 & 0.29 & $<0.06$ & 3,690 & 0.03 & 7.1 & 1 & 17.6 & 1.1 \\
\hline $05 U-2 / 0-1$ & $<2$ & 56,600 & 19.1 & 504 & 1.4 & 0.62 & 5,460 & 0.62 & 58.4 & 6.5 & 41.7 & 4.7 \\
\hline $05 U-2 / 1-2$ & $<2$ & 55,400 & 19.1 & 501 & 1.4 & 0.67 & 4,900 & 0.61 & 50.9 & 6.5 & 44 & 4.6 \\
\hline $05 U-2 / 2-5$ & $<2$ & 55,300 & 18 & 498 & 1.4 & 0.67 & 3,950 & 0.48 & 47.6 & 5.6 & 41.9 & 4.4 \\
\hline 05U-2/Bedrock & $<2$ & 18,400 & 4.7 & 347 & 0.36 & $<0.06$ & 390 & 0.009 & 7.2 & 0.65 & 10.5 & 1.2 \\
\hline 21/0-1 & $<2$ & 38,600 & 3.3 & 579 & 1.1 & 0.21 & 25,200 & 0.33 & 45 & 4.2 & 35.1 & 2.2 \\
\hline $21 / 1-2$ & $<2$ & 39,100 & 3.1 & 601 & 1 & 0.2 & 23,500 & 0.19 & 44.9 & 4 & 32.6 & 2.2 \\
\hline $21 / 2-5$ & $<2$ & 38,400 & 3.5 & 591 & 1.1 & 0.21 & 21,200 & 0.16 & 39.1 & 3.8 & 33.2 & 2.1 \\
\hline 21/Bedrock & $<2$ & 15,900 & $<1$ & 267 & 0.35 & $<0.06$ & 7,680 & 0.01 & 9.4 & 0.81 & 14.2 & 0.81 \\
\hline
\end{tabular}


Table 5. Geochemical data.-Continued

ICP-MS data-continued.

\begin{tabular}{|c|c|c|c|c|c|c|c|c|c|c|c|c|}
\hline Sample no. & $\begin{array}{c}\mathrm{Cu} \\
(\mathrm{ppm})\end{array}$ & $\begin{array}{l}\mathrm{Fe} \\
(\mathrm{ppm})\end{array}$ & $\begin{array}{c}\mathrm{Ga} \\
(\mathrm{ppm})\end{array}$ & $\begin{array}{c}\mathrm{K} \\
(\mathrm{ppm})\end{array}$ & $\begin{array}{c}\text { La } \\
(p p m)\end{array}$ & $\begin{array}{c}\mathrm{Li} \\
(\mathrm{ppm})\end{array}$ & $\begin{array}{c}\text { Mg } \\
(p p m)\end{array}$ & $\begin{array}{c}\mathrm{Mn} \\
(\mathrm{ppm})\end{array}$ & $\begin{array}{c}\text { Mo } \\
\text { (ppm) }\end{array}$ & $\begin{array}{c}\mathrm{Na} \\
(\mathrm{ppm})\end{array}$ & $\begin{array}{c}\mathrm{Nb} \\
\text { (ppm) }\end{array}$ & $\begin{array}{c}\mathrm{Ni} \\
\text { (ppm) }\end{array}$ \\
\hline $493 / 0-1$ & 38.3 & 31,100 & 17.5 & 24,500 & 124 & 31.2 & 11,100 & 605 & 1.1 & 15,000 & 19 & 19.3 \\
\hline 493/1-2 & 32.8 & 33,600 & 18.7 & 26,800 & 98.4 & 35.6 & 12,100 & 625 & 1.2 & 14,900 & 18 & 21.7 \\
\hline $493 / 2-5$ & 29.4 & 29,300 & 16.2 & 24,200 & 50.1 & 33.6 & 10,100 & 523 & 1 & 11,400 & 15 & 19.4 \\
\hline 493/Bedrock & 11.1 & 11,000 & 7.5 & 16,000 & 16.7 & 28 & 9,020 & 150 & 0.46 & 6,520 & 4.9 & 10.8 \\
\hline 29/0-1 & 35.3 & 26,000 & 16.5 & 37,300 & 43.7 & 42.2 & 10,800 & 417 & 1.2 & 10,900 & 18 & 18.3 \\
\hline $29 / 1-2$ & 32.9 & 26,000 & 17.1 & 37,900 & 43.7 & 46 & 9,990 & 386 & 1 & 10,700 & 18 & 18.4 \\
\hline $29 / 2-5$ & 24.1 & 21,400 & 16.7 & 37,600 & 36.4 & 52.4 & 8,010 & 285 & 0.86 & 6,820 & 16 & 16.5 \\
\hline $55 / 0-1$ & 40.6 & 24,400 & 12.1 & 24,300 & 47.2 & 27 & 14,400 & 499 & 1.3 & 8,980 & 13 & 16 \\
\hline $55 / 1-2$ & 39.4 & 29,200 & 14.8 & 28,700 & 39.8 & 34.4 & 13,400 & 399 & 1.4 & 8,210 & 14 & 18.4 \\
\hline $55 / 2-5$ & 32.1 & 27,200 & 14.3 & 28,000 & 37.5 & 32.5 & 11,200 & 333 & 1.2 & 7,710 & 14 & 17.5 \\
\hline 55/Bedrock & 8 & 4,050 & 2.6 & 16,300 & 3.5 & 4.2 & 669 & 11.1 & 0.22 & 394 & 1 & 1.9 \\
\hline 54/0-1 & 45.3 & 24,300 & 12.9 & 25,800 & 41.3 & 28.6 & 11,100 & 451 & 1.7 & 7,990 & 13 & 15.8 \\
\hline $54 / 1-2$ & 44.2 & 32,500 & 17.5 & 33,200 & 34.8 & 38.8 & 11,500 & 343 & 2.4 & 5,480 & 18 & 19.6 \\
\hline $54 / 2-5$ & 55.3 & 27,300 & 15.2 & 29,700 & 30.4 & 35 & 9,520 & 280 & 2.1 & 4,240 & 13 & 16.6 \\
\hline 54/Bedrock & 10.4 & 7,280 & 2.8 & 17,100 & 6.6 & 6.6 & 721 & 131 & 2.9 & 394 & 1.1 & 4.9 \\
\hline $53 / 0-1$ & 32.1 & 17,600 & 12.9 & 36,500 & 25.6 & 30.4 & 5,660 & 332 & 1 & 8,460 & 12 & 12.7 \\
\hline $53 / 2-5$ & 35.9 & 20,500 & 17.5 & 33,500 & 36.4 & 47.6 & 5,610 & 304 & 1.1 & 7,480 & 18 & 14.7 \\
\hline 53/Bedrock & 6.7 & 4,010 & 3.3 & 6,800 & 5 & 12.8 & 556 & 113 & 0.4 & 265 & 1.4 & 4.3 \\
\hline 51/0-1 & 36.6 & 23,700 & 12.3 & 28,600 & 31.4 & 30.6 & 6,810 & 636 & 1.7 & 6,380 & 13 & 17.1 \\
\hline $51 / 1-2$ & 32.3 & 23,300 & 12.3 & 28,600 & 30.8 & 30.9 & 6,670 & 648 & 1.7 & 6,020 & 13 & 16.9 \\
\hline $51 / 2-5$ & 32.4 & 24,400 & 12.8 & 29,000 & 33.1 & 32.3 & 7,220 & 675 & 1.7 & 5,920 & 13 & 18.5 \\
\hline 51/Bedrock & 2.1 & 1,290 & 0.7 & 2,140 & 2 & 4.8 & 5,020 & 312 & 0.22 & 230 & 0.44 & 4.2 \\
\hline 50/0-1 & 30.6 & 16,900 & 8 & 26,200 & 26.8 & 15.7 & 4,970 & 725 & 1 & 4,330 & 7.1 & 11.7 \\
\hline $50 / 1-2$ & 22.1 & 17,000 & 7.3 & 25,000 & 28.6 & 14.7 & 4,510 & 719 & 0.85 & 4,140 & 6.2 & 11.1 \\
\hline $50 / 2-5$ & 24 & 17,000 & 7.4 & 24,800 & 26.4 & 15.3 & 4,560 & 721 & 0.99 & 4,140 & 6 & 11.8 \\
\hline 50/Bedrock & 6.2 & 3,560 & 1 & 5,940 & 2.8 & 4.8 & 431 & 363 & 0.4 & 168 & 0.3 & 3.9 \\
\hline 49/0-1 & 30.1 & 13,600 & 9 & 27,300 & 29.9 & 21.5 & 7,020 & 346 & 0.64 & 4,860 & 8 & 9.2 \\
\hline $49 / 1-2$ & 24.6 & 11,600 & 8.2 & 25,400 & 24.7 & 19.4 & 5,740 & 285 & 0.63 & 3,780 & 7.1 & 8.4 \\
\hline $49 / 2-5$ & n.d. & n.d. & n.d. & n.d. & n.d. & n.d. & n.d. & n.d. & n.d. & n.d. & n.d. & n.d. \\
\hline 49/Bedrock & 5.2 & 1,520 & 1.4 & 4,330 & 3.5 & 8.3 & 237 & 17.9 & 0.2 & 146 & 0.56 & 1.7 \\
\hline $48 / 0-1$ & 21.2 & 13,000 & 10.5 & 16,500 & 26.2 & 25 & 5,680 & 386 & 1 & 5,970 & 7.9 & 8 \\
\hline $48 / 1-2$ & 18.6 & 11,800 & 9.7 & 16,500 & 26.1 & 23.2 & 5,080 & 366 & 0.98 & 5,970 & 6.5 & 7.4 \\
\hline $48 / 2-5$ & 18.9 & 11,800 & 10.1 & 15,600 & 29.2 & 24.1 & 4,900 & 345 & 1 & 5,420 & 7.8 & 7.2 \\
\hline $47 / 2-5$ & 40.3 & 19,200 & 15.8 & 28,700 & 29.4 & 53.2 & 5,790 & 242 & 0.75 & 7,280 & 16 & 15 \\
\hline 47/Bedrock & 35.4 & 38,800 & 3.5 & 8,240 & 4.4 & 12.7 & 777 & 218 & 0.72 & 363 & 1.2 & 6.8 \\
\hline $30 / 0-1$ & 28.1 & 18,800 & 16.2 & 23,800 & 32.6 & 45.8 & 4,610 & 171 & 0.81 & 4,020 & 13 & 13.2 \\
\hline $30 / 1-2$ & 37.9 & 19,600 & 18.6 & 23,200 & 33.7 & 54.6 & 4,230 & 141 & 0.87 & 3,140 & 14 & 14.1 \\
\hline $30 / 2-5$ & 37.2 & 17,000 & 19.8 & 21,500 & 28.7 & 59.2 & 3,730 & 88 & 0.73 & 1,960 & 12 & 13.1 \\
\hline 30/Bedrock & 5.2 & 4,890 & 3.4 & 9,180 & 7.9 & 9.5 & 509 & 31.1 & 0.37 & 328 & 1.6 & 2.9 \\
\hline 46/0-1 & n.d. & n.d. & n.d. & n.d. & n.d. & n.d. & n.d. & n.d. & n.d. & n.d. & n.d. & n.d. \\
\hline $46 / 1-2$ & n.d. & n.d. & n.d. & n.d. & n.d. & n.d. & n.d. & n.d. & n.d. & n.d. & n.d. & n.d. \\
\hline $46 / 2-5$ & n.d. & n.d. & n.d. & n.d. & n.d. & n.d. & n.d. & n.d. & n.d. & n.d. & n.d. & n.d. \\
\hline 46/Bedrock & n.d. & n.d. & n.d. & n.d. & n.d. & n.d. & n.d. & n.d. & n.d. & n.d. & n.d. & n.d. \\
\hline 45/0-1 & 25.1 & 10,200 & 7.6 & 32,100 & 15.4 & 16 & 2,490 & 192 & 0.51 & 2,570 & 7.2 & 7.8 \\
\hline $45 / 1-2$ & 12.4 & 6,900 & 6 & 27,800 & 9.6 & 12.7 & 1,680 & 142 & 0.32 & 1,600 & 4.1 & 5.7 \\
\hline $45 / 2-5$ & 34.6 & 9,590 & 8.1 & 34,600 & 15.4 & 17.1 & 2,430 & 169 & 0.48 & 2,340 & 7.9 & 7.9 \\
\hline 45/Bedrock & 4.3 & 2,210 & 3.4 & 13,800 & 4.7 & 11.4 & 927 & 21.1 & 0.2 & 460 & 1.6 & 3.3 \\
\hline $44 / 0-1$ & 20.7 & 11,800 & 8.6 & 25,400 & 25.4 & 27.2 & 9,070 & 266 & 0.56 & 5,550 & 6.8 & 9.4 \\
\hline $44 / 1-2$ & 18.6 & 13,400 & 9.4 & 26,000 & 31.6 & 28.3 & 10,500 & 298 & 0.57 & 6,340 & 7.9 & 9.8 \\
\hline $44 / 2-5$ & 23.1 & 13,100 & 9 & 25,400 & 30.6 & 27.6 & 9,970 & 281 & 0.59 & 6,260 & 9.9 & 9.7 \\
\hline 44/Bedrock & 8 & 1,910 & 3.2 & 12,400 & 6.4 & 22.5 & 830 & 9.4 & 0.1 & 341 & 2.3 & 2.7 \\
\hline $05 U-1 / 0-1$ & 32.6 & 21,700 & 12.4 & 32,900 & 41 & 27.7 & 7,870 & 369 & 1 & 5,610 & 19 & 16.6 \\
\hline $05 U-1 / 1-2$ & 31.4 & 17,200 & 10.8 & 36,800 & 29 & 23.6 & 6,200 & 290 & 0.72 & 4,340 & 11 & 14 \\
\hline 05U-1/Bedrock & 4.2 & 2,600 & 2.6 & 15,400 & 4 & 4.5 & 688 & 119 & 0.2 & 454 & 1.4 & 2.8 \\
\hline $05 U-2 / 0-1$ & 33.2 & 20,000 & 11.3 & 30,300 & 29.7 & 29.7 & 6,930 & 367 & 0.89 & 5,950 & 12 & 15.7 \\
\hline $05 U-2 / 1-2$ & 30.5 & 20,000 & 11.1 & 30,500 & 27.3 & 28.1 & 6,690 & 367 & 0.93 & 6,020 & 14 & 15.8 \\
\hline $05 U-2 / 2-5$ & 24.4 & 18,000 & 10.5 & 32,400 & 24.6 & 26.7 & 6,120 & 310 & 0.75 & 5,580 & 10 & 14.1 \\
\hline 05U-2/Bedrock & 3.4 & 1,970 & 2.9 & 19,600 & 4.1 & 6 & 650 & 34.2 & 0.09 & 547 & 1.1 & 2.2 \\
\hline $21 / 0-1$ & 18.7 & 14,800 & 7.7 & 19,300 & 23.6 & 21.1 & 7,210 & 345 & 0.51 & 6,630 & 5.2 & 8.4 \\
\hline $21 / 1-2$ & 16.8 & 14,000 & 7.8 & 19,800 & 22.9 & 20.5 & 6,820 & 327 & 0.44 & 6,690 & 5.3 & 7.9 \\
\hline $21 / 2-5$ & 20.1 & 13,700 & 7.4 & 20,100 & 20 & 20.3 & 6,200 & 309 & 0.43 & 6,720 & 5.7 & 7.7 \\
\hline 21/Bedrock & 3.9 & 3,380 & 3 & 11,300 & 5.3 & 9.7 & 1,560 & 49.4 & 0.2 & 750 & 1.8 & 2 \\
\hline
\end{tabular}


Table 5. Geochemical data.-Continued

ICP-MS data-continued.

\begin{tabular}{|c|c|c|c|c|c|c|c|c|c|c|c|c|c|}
\hline Sample no. & $\begin{array}{c}P \\
(p p m)\end{array}$ & $\begin{array}{c}\mathrm{Pb} \\
(\mathrm{ppm})\end{array}$ & $\begin{array}{c}\mathbf{R b} \\
(\mathrm{ppm})\end{array}$ & $\begin{array}{c}\mathrm{Sb} \\
(\mathrm{ppm})\end{array}$ & $\begin{array}{c}\text { Sc } \\
\text { (ppm) }\end{array}$ & $\begin{array}{c}\mathrm{Sr} \\
(\mathrm{ppm})\end{array}$ & $\begin{array}{c}\text { Th } \\
\text { (ppm) }\end{array}$ & $\begin{array}{c}\mathrm{Ti} \\
\text { (ppm) }\end{array}$ & $\begin{array}{c}\mathrm{TI} \\
\text { (ppm) }\end{array}$ & $\begin{array}{c}\mathbf{U} \\
(\mathrm{ppm})\end{array}$ & $\begin{array}{c}\mathrm{V} \\
(\mathrm{ppm})\end{array}$ & $\begin{array}{c}\mathrm{Y} \\
\text { (ppm) }\end{array}$ & $\begin{array}{c}\mathrm{Zn} \\
(\mathrm{ppm})\end{array}$ \\
\hline $493 / 0-1$ & 1,000 & 45.6 & 104 & 1.9 & 11.1 & 371 & 14.7 & 4,880 & 0.68 & 2.62 & 83.6 & 26.5 & 96.7 \\
\hline $493 / 1-2$ & 998 & 43.2 & 113 & 2 & 12 & 384 & 15.5 & 4,970 & 0.74 & 2.79 & 90.7 & 28 & 102 \\
\hline $493 / 2-5$ & 771 & 29.7 & 103 & 1.9 & 10.3 & 316 & 12.7 & 3,840 & 0.7 & 2.2 & 80 & 23.5 & 84.6 \\
\hline 493/Bedrock & 405 & 12.4 & 58.1 & 1.1 & 3.7 & 409 & 3.8 & 1,270 & 0.44 & 0.89 & 30.6 & 7.9 & 30 \\
\hline $29 / 0-1$ & 718 & 46.3 & 141 & 1.3 & 8.8 & 253 & 12.1 & 3,890 & 0.87 & 2.36 & 65.1 & 21.9 & 71.2 \\
\hline $29 / 1-2$ & 692 & 37.3 & 146 & 1.2 & 8.9 & 255 & 12.9 & 3,920 & 0.88 & 2.56 & 65 & 22.4 & 65.5 \\
\hline $29 / 2-5$ & 368 & 28.3 & 157 & 0.93 & 7.5 & 207 & 10.1 & 3,320 & 0.94 & 2.15 & 54.6 & 17.7 & 45.9 \\
\hline 29/Bedrock & 64 & 6.42 & 35.6 & 0.2 & 1 & 30.5 & 1.3 & 309 & 0.23 & 0.24 & 4.8 & 2.9 & 3.7 \\
\hline 55/0-1 & 824 & 46.5 & 89.8 & 1.1 & 8 & 236 & 12.8 & 3,520 & 0.5 & 2.51 & 58.7 & 22.5 & 65.9 \\
\hline $55 / 1-2$ & 911 & 30 & 109 & 1.1 & 9.5 & 245 & 12.8 & 3,980 & 0.56 & 2.55 & 66.5 & 24.2 & 67.2 \\
\hline $55 / 2-5$ & 758 & 21.2 & 107 & 0.99 & 9.1 & 231 & 12.9 & 3,600 & 0.53 & 2.38 & 61.7 & 23.5 & 58 \\
\hline 55/Bedrock & 148 & 6.46 & 45.8 & 0.2 & 0.8 & 43.8 & 0.87 & 190 & 0.24 & 0.19 & 3.1 & 2 & $<3$ \\
\hline $54 / 0-1$ & 839 & 45.2 & 91.6 & 1.4 & 7.8 & 342 & 10.2 & 3,150 & 0.53 & 2.07 & 57.8 & 20.1 & 67.5 \\
\hline $54 / 1-2$ & 1,170 & 36.2 & 120 & 1.6 & 10.5 & 716 & 12 & 3,580 & 0.63 & 2.46 & 73.5 & 24.9 & 69.5 \\
\hline $54 / 2-5$ & 967 & 28.5 & 107 & 1.3 & 8.9 & 649 & 10.4 & 2,680 & 0.57 & 2.51 & 61.4 & 21.7 & 57.9 \\
\hline 54/Bedrock & 566 & 8.22 & 37.4 & 0.28 & 2.2 & 351 & 1.23 & 214 & 0.21 & 0.55 & 17.4 & 3.9 & 10.3 \\
\hline $53 / 0-1$ & 559 & 46.4 & 137 & 1.1 & 5.6 & 222 & 8.47 & 2,730 & 0.86 & 1.86 & 45.1 & 15 & 69 \\
\hline $53 / 2-5$ & 382 & 33.8 & 130 & 1.3 & 7.4 & 243 & 11.8 & 4,200 & 0.86 & 2.5 & 61.8 & 18.4 & 58.9 \\
\hline 53/Bedrock & 128 & 9.58 & 25.4 & 0.54 & 1 & 66 & 1.76 & 393 & 0.18 & 0.26 & 7.7 & 3.1 & 12 \\
\hline 51/0-1 & 577 & 34.5 & 98.5 & 1.1 & 8.2 & 130 & 11.2 & 3,530 & 0.74 & 2.58 & 58.2 & 22.4 & 74.5 \\
\hline $51 / 1-2$ & 549 & 32.2 & 97.8 & 1.1 & 8 & 124 & 10.5 & 3,360 & 0.76 & 2.48 & 56.4 & 21.2 & 75.9 \\
\hline $51 / 2-5$ & 520 & 31.9 & 100 & 1.1 & 8.5 & 129 & 12.6 & 3,360 & 0.79 & 2.63 & 59.7 & 23 & 75.3 \\
\hline 51/Bedrock & 108 & 1.14 & 5.3 & 0.08 & 0.6 & 217 & 0.42 & 126 & $<0.08$ & 0.44 & 5.5 & 2 & 6.5 \\
\hline 50/0-1 & 658 & 35 & 75.1 & 0.58 & 4.9 & 94.8 & 7.38 & 3,110 & 0.42 & 1.96 & 39.3 & 19.4 & 49.6 \\
\hline $50 / 1-2$ & 570 & 37 & 70.4 & 0.49 & 4.7 & 87.4 & 8.98 & 3,270 & 0.4 & 2.26 & 38.7 & 20.5 & 43.8 \\
\hline $50 / 2-5$ & 569 & 39.1 & 70.4 & 0.53 & 4.9 & 86.3 & 8.09 & 3,320 & 0.41 & 2.12 & 39.5 & 20.4 & 46.9 \\
\hline 50/Bedrock & 76 & 3.05 & 15.8 & 0.1 & 0.5 & 24.4 & 0.76 & 111 & 0.08 & 0.19 & 2.6 & 3.2 & 4.1 \\
\hline 49/0-1 & 642 & 31.6 & 88.6 & 0.66 & 5.2 & 125 & 11 & 3,040 & 0.62 & 3.32 & 49.3 & 22.6 & 45.5 \\
\hline $49 / 1-2$ & 506 & 27.9 & 81.8 & 0.6 & 4.6 & 107 & 8.75 & 2,510 & 0.56 & 2.71 & 43.2 & 19.3 & 39 \\
\hline $49 / 2-5$ & n.d. & n.d. & n.d. & n.d. & n.d. & n.d. & n.d. & n.d. & n.d. & n.d. & n.d. & n.d. & n.d. \\
\hline 49/Bedrock & 58 & 4.38 & 13.1 & 0.2 & 0.4 & 21.4 & 1.09 & 210 & 0.09 & 0.47 & 13 & 1.9 & 4.2 \\
\hline 48/0-1 & 601 & 20.6 & 56.6 & 0.66 & 5.9 & 113 & 10.4 & 2,930 & 0.56 & 2.45 & 43.8 & 20 & 34.9 \\
\hline $48 / 1-2$ & 573 & 18.3 & 54.3 & 0.62 & 5.5 & 115 & 9.92 & 2,710 & 0.52 & 2.27 & 39.7 & 19.4 & 30.2 \\
\hline $48 / 2-5$ & 552 & 17.8 & 50.4 & 0.74 & 5.6 & 133 & 12 & 2,940 & 0.52 & 2.48 & 40.7 & 21 & 29 \\
\hline 48/Bedrock & 146 & 19.5 & 2.8 & 3.2 & 2 & 63 & 8.87 & 1,430 & 1.67 & 1.78 & 116 & 6.2 & 13.5 \\
\hline 47/Bedrock & 392 & 15.9 & 28.7 & 0.96 & 2.2 & 38 & 4.35 & 284 & 0.21 & 1.58 & 240 & 10 & 21.3 \\
\hline $30 / 0-1$ & 592 & 29.3 & 80.9 & 0.88 & 6.1 & 251 & 9.01 & 3,130 & 0.66 & 3.4 & 52.7 & 17.9 & 49.9 \\
\hline $30 / 1-2$ & 606 & 29.5 & 76.9 & 0.84 & 6.8 & 301 & 8.98 & 3,020 & 0.68 & 3.94 & 56.9 & 17 & 57.2 \\
\hline $30 / 2-5$ & 599 & 24.7 & 73.2 & 0.67 & 6.2 & 378 & 6.4 & 2,440 & 0.71 & 3.96 & 53.8 & 14.5 & 43.3 \\
\hline 30/Bedrock & 115 & 11 & 31.8 & 0.21 & 1 & 98.2 & 1.54 & 410 & 0.23 & 0.53 & 6.8 & 3 & 5.5 \\
\hline 46/0-1 & n.d. & n.d. & n.d. & n.d. & n.d. & n.d. & n.d. & n.d. & n.d. & n.d. & n.d. & n.d. & n.d. \\
\hline $46 / 1-2$ & n.d. & n.d. & n.d. & n.d. & n.d. & n.d. & n.d. & n.d. & n.d. & n.d. & n.d. & n.d. & n.d. \\
\hline $46 / 2-5$ & n.d. & n.d. & n.d. & n.d. & n.d. & n.d. & n.d. & n.d. & n.d. & n.d. & n.d. & n.d. & n.d. \\
\hline 46/Bedrock & n.d. & n.d. & n.d. & n.d. & n.d. & n.d. & n.d. & n.d. & n.d. & n.d. & n.d. & n.d. & n.d. \\
\hline 45/0-1 & 326 & 26.3 & 97.3 & 0.77 & 4.3 & 110 & 5.31 & 2,700 & 0.58 & 1.82 & 40.1 & 18 & 28.2 \\
\hline $45 / 1-2$ & 207 & 20.3 & 84.8 & 0.57 & 3 & 83.3 & 3.04 & 1,910 & 0.5 & 1.16 & 28.5 & 11.9 & 18.8 \\
\hline $45 / 2-5$ & 293 & 24.9 & 103 & 0.81 & 4.5 & 111 & 5.22 & 2,840 & 0.6 & 1.81 & 40.7 & 19.3 & 27.8 \\
\hline 45/Bedrock & 64 & 7.52 & 43.8 & 0.2 & 1.2 & 36.3 & 1.15 & 389 & 0.26 & 0.26 & 8.9 & 2.9 & 7 \\
\hline $44 / 0-1$ & 574 & 18.8 & 77.1 & 0.43 & 4.7 & 134 & 8.67 & 2,230 & 0.47 & 2.11 & 31.7 & 18.4 & 35.6 \\
\hline $44 / 1-2$ & 690 & 17.7 & 79.2 & 0.48 & 5.1 & 148 & 10.5 & 2,660 & 0.47 & 2.4 & 36.1 & 21.3 & 38.5 \\
\hline $44 / 2-5$ & 659 & 16.6 & 74.7 & 0.47 & 4.9 & 143 & 10.3 & 2,700 & 0.46 & 2.46 & 35.3 & 20.6 & 36.8 \\
\hline 44/Bedrock & 87 & 4.82 & 35.4 & 0.1 & 0.8 & 68.2 & 2.04 & 758 & 0.2 & 0.31 & 7 & 3.5 & 7.1 \\
\hline $05 U-1 / 0-1$ & 794 & 34.3 & 94 & 0.99 & 7.7 & 169 & 13.9 & 4,230 & 0.64 & 3.24 & 58.5 & 25.1 & 75.1 \\
\hline $05 U-1 / 1-2$ & 646 & 30.4 & 92.4 & 0.84 & 6.2 & 140 & 9.54 & 2,780 & 0.62 & 2.33 & 47 & 17.6 & 71.3 \\
\hline 05U-1/Bedrock & 64 & 6.58 & 43.7 & 0.26 & 1.5 & 34.5 & 0.92 & 368 & 0.28 & 0.22 & 6.5 & 2.5 & 13 \\
\hline $05 U-2 / 0-1$ & 698 & 45.6 & 91 & 0.87 & 7.4 & 171 & 9.58 & 3,370 & 0.6 & 2.67 & 55.4 & 20.2 & 73.7 \\
\hline $05 U-2 / 1-2$ & 656 & 47 & 89.7 & 0.87 & 7 & 169 & 8.91 & 3,050 & 0.62 & 2.42 & 52.1 & 18.5 & 72.1 \\
\hline $05 U-2 / 2-5$ & 308 & 46.4 & 92.5 & 0.72 & 6.5 & 154 & 8 & 2,630 & 0.64 & 2.13 & 47.8 & 15.7 & 53.8 \\
\hline 05U-2/Bedrock & 53 & 8.18 & 52.1 & 0.2 & 0.9 & 54.6 & 0.88 & 276 & 0.34 & 0.23 & 5.3 & 1.8 & 5.6 \\
\hline 21/0-1 & 449 & 19.4 & 65.2 & 0.41 & 4.3 & 131 & 7.73 & 2,350 & 0.42 & 2.16 & 37.1 & 16.9 & 43.8 \\
\hline $21 / 1-2$ & 414 & 17.9 & 66.1 & 0.42 & 4 & 122 & 7.41 & 2,290 & 0.42 & 2.01 & 34.7 & 16.5 & 33 \\
\hline $21 / 2-5$ & 393 & 16.1 & 67.2 & 0.41 & 3.8 & 115 & 6.26 & 2,340 & 0.41 & 1.87 & 33.4 & 15.7 & 33.7 \\
\hline 21/Bedrock & 122 & 5.6 & 35.4 & 0.2 & 0.9 & 35.4 & 1.42 & 484 & 0.22 & 0.55 & 7.4 & 3.6 & 7.2 \\
\hline
\end{tabular}

